Research Article

Lorenzo D’Ambrosio and Enzo Mitidieri*

\title{
Entire solutions of certain fourth order elliptic problems and related inequalities
}

https://doi.org/10.1515/anona-2021-0217

Received November 16, 2021; accepted November 21, 2021.

Abstract: We study distributional solutions of semilinear biharmonic equations of the type

$$
\Delta^{2} u+f(u)=0 \quad \text { on } \mathbb{R}^{N},
$$

where $f$ is a continuous function satisfying $f(t) t \geq c|t|^{q+1}$ for all $t \in \mathbb{R}$ with $c>0$ and $q>1$. By using a new approach mainly based on careful choice of suitable weighted test functions and a new version of HardyRellich inequalities, we prove several Liouville theorems independently of the dimension $N$ and on the sign of the solutions.

Keywords: Liouville theorems, biharmonic operator, Hardy-Rellich inequalities

MSC: 35G20, 31B30, 35B53, 26D10

Dedicated to the memory of our dear friend Stanislav Pohozaev

\begin{tabular}{|c|c|}
\hline 1 & Introduction -786 \\
\hline 2 & A detour on the second order case: the quadratic form approach $\longrightarrow 789$ \\
\hline 3 & Some simple results on biharmonic problems $\longrightarrow 791$ \\
\hline 4 & On the notion of solution: other related problems -793 \\
\hline 5 & Asymptotic Hardy-Rellich type inequalities $\longrightarrow 795$ \\
\hline 5.1 & An integral identity $-\mathbf{8 0 0}$ \\
\hline 5.2 & Some functional weighted quadratic inequalities $-\mathbf{8 0 2}$ \\
\hline 5.2 .1 & Some results on functional positive quadratic forms $-\mathbf{8 0 4}$ \\
\hline 6 & A priori estimates and Liouville theorems $-\mathbf{8 0 7}$ \\
\hline 6.1 & A priori estimates $-\mathbf{8 0 7}$ \\
\hline 6.2 & Some glimpses on Liouville theorems: weak solutions $-\mathbf{8 1 0}$ \\
\hline 6.3 & Weighted a priori estimates $-\mathbf{8 1 4}$ \\
\hline 6.4 & Distributional solutions: a priori estimates $\longrightarrow \mathbf{8 1 6}$ \\
\hline 6.5 & Liouville theorems: distributional solutions $-\mathbf{8 1 8}$ \\
\hline 6.6 & A generalization $-\mathbf{8 2 1}$ \\
\hline 7 & Further remarks and results on the solutions $-\mathbf{8 2 2}$ \\
\hline 7.1 & Representation formula for $u^{2}-\mathbf{8 2 2}$ \\
\hline 7.2 & Remarks on the sign of the solutions -825 \\
\hline 7.3 & Uniqueness -825 \\
\hline A & Representation formula $-\mathbf{8 2 7}$ \\
\hline
\end{tabular}

Lorenzo D’Ambrosio, Dipartimento di Matematica, Università degli Studi di Bari Aldo Moro, via E.Orabona, 4, I-70125 Bari, E-mail: lorenzo.dambrosio@uniba.it

*Corresponding Author: Enzo Mitidieri, Dipartimento di Matematica e Geoscienze, Università degli Studi di Trieste, via A.Valerio, 12/1, I-34127 Trieste, E-mail: mitidier@units.it 


\section{Introduction}

In a well known work Brezis [5] proved the following result.

Theorem 1.1. Let $q>1$. If $u \in L_{\text {loc }}^{q}\left(\mathbb{R}^{N}\right)$ is a distributional solution of

$$
\Delta u \geq|u|^{q-1} u \text { on } \mathbb{R}^{N},
$$

then $u \leq 0$ a.e. in $\mathbb{R}^{N}$.

In particular this implies.

Theorem 1.2. Let $q>1$. If $u \in L_{\text {loc }}^{q}\left(\mathbb{R}^{N}\right)$ is a distributional solution of

$$
\Delta u=|u|^{q-1} u \quad \text { on } \mathbb{R}^{N},
$$

then $u \equiv 0$ a.e. in $\mathbb{R}^{N}$.

The interesting point here, besides the quite general functional framework, is that no assumptions on the behavior nor on the sign of the possible solutions of (1.2) are made. Brezis's technique is based on the following form of Kato inequality (see [5, Lemma A.1]),

$$
u, f \in L_{l o c}^{1}(\Omega) \text { such that } \Delta u \geq f \text {, then } \Delta u^{+} \geq \operatorname{sign}^{+}(u) f,
$$

and on a construction of a suitable barrier function. These tools are typically second order in nature, so, in general it is hopeless to use them when dealing with problems of order higher than two. Comprehensive results in the Brezis' spirit for quasilinear elliptic inequalities of second order on $\mathbb{R}^{N}$ have been obtained in a series of papers by Farina and Serrin [15, 16] and the Authors [10-12]. In addition these results are also studied in the subelliptic framework $[12,13]$ and in the Riemannian setting [4].

These results suggest a general natural problem for higher order elliptic equations and inequalities.

General problem: What are the necessary conditions that guarantee the existence of non trivial solutions for higher order nonlinear elliptic coercive ${ }^{1}$ problems on $\mathbb{R}^{N}$ ?

The results for the second order case cited above, altogether are proved in the spirit of [27]. For higher order problem the beneficial of a systematic approach for studying coercive elliptic problems is still missing. The aim of this paper is to give a contribution to develop a possible unitary method for higher order elliptic equation and inequalities of coercive type and it represents a first step in this direction.

In concrete situations for fourth order semilinear elliptic equations with simple power nonlinearities, the problem is connected to find a so called critical exponent. Here, by critical exponent we mean the existence of $q^{\star}(N)>1$, depending on the dimension $N$ such that there are no non trivial solutions for $q<q^{\star}(N)$ and there exist non trivial solutions for $q>q^{\star}(N)$. Then from Theorem 1.2 we can say that for equation (1.2) the critical exponent is $q^{\star}(N)=\infty$.

Let us consider the fourth order analogue of (1.2), that is

$$
-\Delta^{2} u=|u|^{q-1} u \quad \text { on } \mathbb{R}^{N}, q>1 .
$$

It is well known that these kind of problems have strong connections with differential geometry [7, 8], higher order Schrödinger equations [20, 24, 31] and models for suspension bridges [17]. In this regard see [19] for further results on related applications of polyharmonic elliptic equations.

Looking at solutions of (1.3) in the natural global space $H^{2}\left(\mathbb{R}^{N}\right)$, it is clear that for $q \leq \frac{N+4}{N-4}$ the only solution $u$ is given by $u \equiv 0$ a.e. in $\mathbb{R}^{N}$. Of course, the interesting problem is when $u$ does not belong to the global space $H^{2}\left(\mathbb{R}^{N}\right)$, so there is no a priori knowledge of the behavior at infinity of the solutions.

1 We call a problem coercive if its formal Euler-Lagrange functional is coercive in its natural functional framework. 
Notice that if in (1.3) we have $q=1$, it is easy to see that the equation admits nontrivial solutions in dimension $N=1$, and hence in any dimension.

It is well known that the literature on nonlinear higher order coercive equations is far from complete. However there exist notable results due to Bernis [2] that for some particular biharmonic problem reads as follows.

Theorem 1.3. Let $u \in H_{\text {loc }}^{2}\left(\mathbb{R}^{N}\right) \cap L_{\text {loc }}^{q+1}\left(\mathbb{R}^{N}\right)$ be a solution of (1.3). If $q(N-4) \leq N+4$, then $u \equiv 0$ a.e. in $\mathbb{R}^{N}$.

A first immediate observation is that from [2] it appears that for (1.3) the Sobolev exponent $\frac{N+4}{N-4}$ is critical (in our sense) when $N>4$. However, and this is the main motivation to write this paper, this is not true.

Even if our main interest is in possible changing sign solution of (1.3), we present here a simple result concerning solutions of (1.3) which do not change sign. Its proof relies on our integral representation results obtained in [6] and it serves as a motivation to focus our attention on the possible sign changing solutions.

Theorem 1.4. Let $u \in L_{\text {loc }}^{q}\left(\mathbb{R}^{N}\right)$ be a distributional solution of (1.3). If $u$ does not change sign, then $u \equiv 0$ a.e. in $\mathbb{R}^{N}$.

More generally we have the following unexpected result for the equation (1.3).

Theorem 1.5. Let $u \in L_{\text {loc }}^{q+1}\left(\mathbb{R}^{N}\right)$ be a distributional solution of (1.3). If

$$
\begin{gathered}
N=1, \ldots, 7, \quad \text { and } q>1, \\
\quad \text { or } \\
N \geq 8 \quad \text { and } 1<q \leq q_{N},
\end{gathered}
$$

where

$$
q_{N}:=\frac{N^{2}+2 N-28+4 \sqrt{4-2 N+2 N^{2}}}{N^{2}-10 N+20},
$$

then $u \equiv 0$ a.e. in $\mathbb{R}^{N}$.

The above result shows that within the class of distributional solutions, if $N \leq 7$ then there is no critical exponent (i.e. $q^{\star}(N)=\infty$ ), while if $N>7$ we have $q_{N}>\frac{N+4}{N-4}$. We believe that the value of $q_{N}$ is not sharp and it can be improved. Indeed we can state the following conjecture.

Conjecture. For any $N \geq 1$ and $q>1$ the only solution of (1.3) is given by $u \equiv 0$ a.e. in $\mathbb{R}^{N}$.

The methods used in this paper apply to more general problems than (1.3). More precisely, some of our results are still valid for distributional solutions of the double inequality

$$
g(u) \geq-\Delta^{2} u \geq f(u), \quad \text { on } \mathbb{R}^{N},
$$

where $f, g \in \mathscr{C}(\mathbb{R})$. Throughout this paper we shall denote by $H$ the following function

$$
H(t):= \begin{cases}f(t) t, & \text { for } t \geq 0, \\ g(t) t, & \text { for } t<0 .\end{cases}
$$

In what follows we shall deal with the autonomous case. The non-autonomous one, that is $f=f(x, u)$ and $g=g(x, u)$ can be studied in similar way. However, for sake of simplicity we limit ourselves to the autonomous case.

We have the following result.

Theorem 1.6. Let $f, g \in \mathscr{C}(\mathbb{R})$ and let $H$ be defined by (1.6). Assume that

$$
H(t) \geq c_{H} \min \left\{|t|^{q+1},|t|^{p+1}\right\}, \quad \forall t \in \mathbb{R} \text {, for some } q \geq p>1
$$


with $c_{H}>0$. Let $u$ be a distributional solution of $\left({ }_{g} \mathbf{P}_{f}\right)$ such that $u \in L_{\text {loc }}^{s}\left(\mathbb{R}^{N}\right), 2 \leq s \leq+\infty$ and $f(u), g(u) \in$ $L_{\text {loc }}^{s^{\prime}}\left(\mathbb{R}^{N}\right)$.

$$
\text { If } N=1, \ldots, 7 \text { or } N \geq 8 \text { and } 1<q \leq q_{N} \text { with } q_{N} \text { defined in (1.5), then } u \equiv 0 \text { a.e. in } \mathbb{R}^{N} \text {. }
$$

Notice that (1.7) is an assumption on the behavior of $f$ and $g$ for nonnegative and nonpositive values of the independent variable, respectively. No assumptions are required on $f$ and $g$ for negative and nonpositive values of the independent variable.

Hypothesis (1.7) allows us to handle nonlinearities that behave differently for positive and negative values of the independent variable or behave differently at the origin and at infinity.

Theorem 1.6 contains several Liouville results for the equation

$$
-\Delta^{2} u=f(u) \text { on } \mathbb{R}^{N} \text {. }
$$

For instance if $f(t)=|t|^{s-1} t+|t|^{p-1} t$ for any $p>1 \geq s>0$, or $f(t)=t e^{|t|}$, or $f(t)=\sinh (t)$, then the above problem has only the trivial solution. Notice that as byproduct, we can deduce that the defocusing Schrödinger equation

$$
i v_{t}+\Delta^{2} v+|v|^{q-1} u=0
$$

has no nontrivial standing wave solutions of the form $v(t, x)=e^{-i \omega^{2} t} u(x)$ with $\omega \in \mathbb{R}$. See [31] and reference therein for further results on this equation, and its connection with several models from physics.

A common feature of the above results is that we do not require any assumption on the behavior of the solutions at infinity. We also point out that for higher order coercive problems Kato's inequality does not hold and in general is not possible to use comparison principles. Thus the main idea to study problems like (1.3) is first to obtain suitable a priori bounds on the various quantities involved in the analysis. These estimates yield a Liouville result for $q$ running in the range of values of Bernis' result i.e. $1<q \leq \frac{N+4}{N-4}$.

To improve this result, i.e. going above the Sobolev exponent for equation (1.3), we develop some machinery by demonstrating functional inequalities related to some quadratic forms. By using the positivity of a particular quadratic form along the solutions of our problems, and taking into account of suitable a priori estimates, we are able to achieve our goal. The involved argument is quite intricate and this is the reason why we begin illustrating the method for the second order prototype equation (1.2) in Section 2.

In Section 3 we study inequalities related to (1.3), obtaining some information on the sign of the possible solution of (1.3). For analogous results see also Section 7.2.

In Section 4 we discuss the different notions of solutions and justify why the study of solutions of $\left({ }_{g} \mathbf{P}_{f}\right)$ is reduced to the study of

$$
-u \Delta^{2} u \geq h, \quad \text { on } \mathbb{R}^{N}
$$

where $h \in L_{\text {loc }}^{1}\left(\mathbb{R}^{N}\right)$.

In Section 5 we develop a number of functional inequalities and positive quadratic forms.

Section 6 is devoted to prove some a priori estimates on the solutions of our problems, which combined with the results of Section 5 yields the Liouville theorems.

Section 7 contains some applications of the results obtained in the preceding sections. In Section 7.1 we prove a special representation formula of $u^{2}$, being $u$ a possible solutions of $\left(\mathbf{P}_{h}\right)$. Section 7.2 deals with some results on the sign of possible solutions of the problem under consideration and their Laplacian. In Section 7.3 we apply our Liouville theorems to the uniqueness problem.

Appendix A recalls a known result on the integral representation of the solutions of some higher order elliptic equation.

Notation. $B_{R}$ will denote the Euclidean ball of radius $R$ centered at the origin $B_{R}:=\{|x| \leq R\}$. By $\omega_{N}$ we denote the measure of the unit Euclidean ball, $\omega_{N}=\left|B_{1}\right|$.

Throughout this paper $\phi_{1}: \mathbb{R} \rightarrow\left[0,+\infty\right.$ [ stands for a standard cut off function, that is $\phi_{1}$ is a smooth function on $\mathbb{R}$ such that $\phi_{1}(t)=1$ for $|t| \leq 1, \phi_{1}(t)=0$ fot $|t| \geq 2$ and $0 \leq \phi_{1}(t) \leq 1$. We set

$$
\phi_{R}(x):=\phi_{1}(|x| / R) \text {, }
$$


the support of $\phi_{R}$ is contained in $B_{2 R}=\{|x| \leq 2 R\}$, while the support of any derivative of $\phi_{R}$ is contained in

$$
A_{R}:=\{R \leq|x| \leq 2 R\} .
$$

Furthermore without loss of generality we shall assume that $\phi_{1}$ is an admissible test function, that is for a fixed $p>1$ there exists $c_{1}>0$ such that

$$
\left\|\frac{\left|\nabla \phi_{1}\right|^{p}}{\phi_{1}^{p-1}}\right\|_{\infty}<c_{1}, \quad\left\|\frac{\left|\Delta \phi_{1}\right|^{p}}{\phi_{1}^{p-1}}\right\|_{\infty}<c_{1}, \quad\left\|\frac{\left|\nabla \Delta \phi_{1}\right|^{p}}{\phi_{1}^{p-1}}\right\|_{\infty}<c_{1} .
$$

Indeed if $\phi_{1}$ is not admissible, then it follows that for large $\gamma, \phi_{1}^{\gamma}$ is admissible.

Finally, in what follows $c$ stands for a positive constant which can vary from line to line and it is independent from the solution $u$ and $R$. Writing $c_{1}$ we always mean a positive constant depending only on the test function $\phi_{1}$, that is $c_{1}=c_{1}\left(\phi_{1}\right)$.

For $N>4$, by $C_{N}$ we denote the normalization positive constant in the relation

$$
\Delta^{2}|x|^{4-N}=C_{N} \delta_{0}
$$

that is $C_{N}:=\frac{1}{4} \Gamma\left(\frac{N-4}{2}\right) \pi^{-N / 2}$.

Finally, in what follows an integral without the indication of the domain of integration, is understood on the whole space $\mathbb{R}^{N}$.

\section{A detour on the second order case: the quadratic form approach}

The purpose of this section is to illustrate a specific method for handling nonexistence theorems for a class of second order coercive equations on $\mathbb{R}^{N}$. For simplicity we restrict our attention to smooth solutions and to the simple prototype equation

$$
\Delta u=|u|^{q-1} u, \quad \text { on } \mathbb{R}^{N} .
$$

The general scheme of our method develops in several steps.

\section{Step 1: Functional identity}

Lemma 2.1. Let $u \in \mathscr{C}^{1}\left(\mathbb{R}^{N}\right), \varphi \in \mathscr{C}_{0}^{2}\left(\mathbb{R}^{N}\right)$ and $v \in \mathscr{C}^{2}\left(\mathbb{R}^{N}\right)$. We have

$$
\int(\nabla u \cdot \nabla(u v \varphi))=\int|\nabla u|^{2} v \varphi-\frac{1}{2} \int u^{2} \Delta v \varphi+E\left(u^{2}, v, \varphi\right),
$$

where

$$
E\left(u^{2}, v, \varphi\right):=-\int u^{2}(\nabla v \cdot \nabla \varphi)-\frac{1}{2} \int u^{2} v \Delta \varphi .
$$

In particular if $\varphi=\phi_{R}$, with $\phi_{R}$ defined in (1.8), and for $k=0,1,\left|v^{(k)}\right| \leq c_{v} R^{\alpha-N-k}$ holds on $A_{R}$, then

$$
\left|E\left(u^{2}, v, \varphi\right)\right| \leq c_{v} c_{1}\left(\phi_{1}\right) R^{\alpha-2} f_{A_{R}}|u|^{2} .
$$

Proof. By computation, we get

$$
\int \nabla u \cdot \nabla(u v \varphi)=\int|\nabla u|^{2} v \varphi+\int(\nabla u \cdot \nabla v) u \varphi+\int(\nabla u \cdot \nabla \varphi) u v .
$$

Next an integrating by parts gives,

$$
\int(\nabla u \cdot \nabla v) u \varphi=-\int(\nabla u \cdot \nabla v) u \varphi-\int u^{2} \Delta v \varphi-\int u^{2}(\nabla v \cdot \nabla \varphi),
$$


that is

$$
\int(\nabla u \cdot \nabla v) u \varphi=-\frac{1}{2} \int u^{2} \Delta v \varphi-\frac{1}{2} \int u^{2}(\nabla v \cdot \nabla \varphi)
$$

Analogously we obtain

$$
\int(\nabla u \cdot \nabla \varphi) u v=-\frac{1}{2} \int u^{2} \Delta \varphi v-\frac{1}{2} \int u^{2}(\nabla v \cdot \nabla \varphi) .
$$

Gluing together these identities, we deduce (2.1).

In order to prove (2.2), we first observe that the domain of integration of the functional $E$ is given by $\operatorname{supp}\left(\nabla \phi_{R}\right)=A_{R}$. Next by using the hypotheses on $v$, we get

$$
\begin{aligned}
\left|E\left(u^{2}, v, \varphi\right)\right| & \leq \int_{A_{R}} u^{2}|\nabla v||\varphi|+\frac{1}{2} \int_{A_{R}} u^{2}|v||\Delta \varphi| \\
& \leq c_{v} R^{\alpha-N-1} c_{1} R^{-1} \int_{A_{R}} u^{2}+\frac{1}{2} c_{v} R^{\alpha-N} c_{1} R^{-2} \int_{A_{R}} u^{2} .
\end{aligned}
$$

We notice that as direct consequence of Lemma 2.1 we deduce that if $v \in \mathscr{C}^{2}\left(\mathbb{R}^{N}\right)$ is nonnegative and superharmonic, then for any nonnegative $\varphi \in \mathscr{C}_{0}^{2}\left(\mathbb{R}^{N}\right)$, the quadratic form,

$$
u \in \mathscr{C}^{1}\left(\mathbb{R}^{N}\right) \mapsto \int(\nabla u \cdot \nabla(u v \varphi))-E\left(u^{2}, v, \varphi\right),
$$

is positive.

\section{Step 2: A functional inequality}

In what follows we set $r:=|x|$ and for $\epsilon>0$,

$$
r_{\epsilon}:=\left(\epsilon^{2}+|\chi|^{2}\right)^{1 / 2}=\left(\epsilon^{2}+r^{2}\right)^{1 / 2},
$$

and for $x \in \mathbb{R}^{N}, \epsilon>0$ and $\alpha \in \mathbb{R}$, we define

$$
v_{\epsilon}(x)=v_{\epsilon}(|x|)=v_{\epsilon}(r):=\frac{1}{\left(\epsilon^{2}+r^{2}\right)^{\frac{N-\alpha}{2}}}=r_{\epsilon}^{\alpha-N} .
$$

We have that $-\Delta v_{\epsilon} \geq 0$ for $N>\alpha \geq 2$. Hence, by choosing $v=v_{\epsilon}$ in Lemma 2.1, we obtain.

Lemma 2.2. Let $N>\alpha \geq 2$. Let $u \in \mathscr{C}^{1}\left(\mathbb{R}^{N}\right)$, and let $\varphi \in \mathscr{C}_{0}^{2}\left(\mathbb{R}^{N}\right)$ be nonnegative. We have

$$
\int\left(\nabla u \cdot \nabla\left(u \frac{\varphi}{r_{\epsilon}^{N-\alpha}}\right)\right) \geq \int|\nabla u|^{2} \frac{\varphi}{r_{\epsilon}^{N-\alpha}}+E\left(u^{2}, r_{\epsilon}^{\alpha-N}, \varphi\right)
$$

and for $\varphi=\phi_{R}$, the estimate (2.2) holds.

Remark 2.3. If $u \in \mathscr{C}_{0}^{1}\left(\mathbb{R}^{N}\right)$, then taking $\varphi=\phi_{R}$ with $R$ large enough, it follows that $E\left(u^{2}, v, \varphi\right)=0$. In addition for any $N \geq \alpha \geq 2$ the quadratic form

$$
u \in \mathscr{C}_{0}^{1}\left(\mathbb{R}^{N}\right) \mapsto \int\left(\nabla u \cdot \nabla \frac{u}{r_{\epsilon}^{N-\alpha}}\right),
$$

is positive. 


\section{Step 3: A priori estimate on the solutions}

Lemma 2.4. Let $q>1$ and let $u \in \mathscr{C}^{2}\left(\mathbb{R}^{N}\right)$ be a solution of

$$
\Delta u=|u|^{q-1} u \text {, on } \mathbb{R}^{N} .
$$

Then

$$
\left(f_{B_{R}} u^{2}\right)^{1 / 2} \leq\left(f_{B_{R}}|u|^{q+1}\right)^{1 / q+1} \leq c R^{-\frac{2}{q-1}}
$$

Proof. Multiplying the equation by $u \phi_{R}$ and using Young inequality we obtain,

$$
\begin{aligned}
& \int|u|^{q+1} \phi_{R}=-\int\left(\nabla u \cdot \nabla\left(u \phi_{R}\right)\right)=-\int|\nabla u|^{2} \phi_{R}-\int u\left(\nabla u \cdot \nabla \phi_{R}\right) \\
& \leq-\int|\nabla u|^{2} \phi_{R}+\frac{1}{2} \int|\nabla u|^{2} \phi_{R}+\frac{1}{2} \int u^{2} \frac{\left|\nabla \phi_{R}\right|^{2}}{\phi_{R}} .
\end{aligned}
$$

By Hölder inequality with exponent $x:=(q+1) / 2$, it follows that

$$
\int|u|^{q+1} \phi_{R} \leq \frac{1}{2} \int u^{2} \frac{\left|\nabla \phi_{R}\right|^{2}}{\phi_{R}} \leq \frac{1}{2}\left(\int|u|^{q+1} \phi_{R}\right)^{1 / x}\left(\int \frac{\left|\nabla \phi_{R}\right|^{2 x^{\prime}}}{\phi_{R}^{x^{\prime}-1}}\right)^{1 / x^{\prime}},
$$

which in turn implies

$$
\int_{B_{R}}|u|^{q+1} \leq \int|u|^{q+1} \phi_{R} \leq 2^{-x^{\prime}} \int \frac{\left|\nabla \phi_{R}\right|^{2 x^{\prime}}}{\phi_{R}^{x^{\prime}-1}} \leq c_{1} R^{N-2 x^{\prime}} .
$$

A simple application of Jensen inequality gives (2.4).

\section{Step 4: A Liouville theorem}

Theorem 2.5. Let $q>1$ and let $u \in \mathscr{C}^{2}\left(\mathbb{R}^{N}\right)$ be a solution of

$$
\Delta u=|u|^{q-1} u \text {, on } \mathbb{R}^{N} .
$$

Then $u \equiv 0$ a.e. in $\mathbb{R}^{N}$.

Proof. Let $N \geq \alpha \geq 2$ and $\varphi=\phi_{R}$. Multiplying the equation by $u \frac{\varphi}{r_{\epsilon}^{N-\alpha}}$, from (2.3) we obtain

$$
\begin{aligned}
0 \geq-\int|u|^{q+1} \frac{\varphi}{r_{\epsilon}^{N-\alpha}} & =\int\left(\nabla u \cdot \nabla\left(u \frac{\varphi}{r_{\epsilon}^{N-\alpha}}\right)\right) \\
& \geq \int|\nabla u|^{2} \frac{\varphi}{r_{\epsilon}^{N-\alpha}}+E\left(u^{2}, r_{\epsilon}^{\alpha-N}, \varphi\right) .
\end{aligned}
$$

Choosing $\alpha=2$, from (2.4) and (2.2) it follows that $\left|E\left(u^{2}, r_{\epsilon}^{2-N}, \varphi\right)\right| \rightarrow 0$ as $R \rightarrow \infty$. Therefore by letting $R \rightarrow+\infty$ in (2.5), we obtain

$$
0 \geq-\int|u|^{q+1} \frac{1}{r_{\epsilon}^{N-2}}=\int\left(\nabla u \cdot \nabla\left(u \frac{1}{r_{\epsilon}^{N-2}}\right)\right) \geq \int|\nabla u|^{2} \frac{1}{r_{\epsilon}^{N-2}} \geq 0 .
$$

This last inequality implies the claim.

\section{Some simple results on biharmonic problems}

Theorem 3.1. Let $q>1$ and let $u \in L_{\text {loc }}^{q}\left(\mathbb{R}^{N}\right)$ be a distributional solution of

$$
-\Delta^{2} u \geq|u|^{q} \quad \text { on } \mathbb{R}^{N} .
$$


1. If $q(N-4) \leq N$, then $u \equiv 0$ a.e. in $\mathbb{R}^{N}$.

2. If $u$ is nontrivial, then $u<0$ a.e. in $\mathbb{R}^{N}$ and $\Delta u \geq 0$ in distributional sense.

Theorem 3.2. Let $q>1$ and let $u \in L_{\text {loc }}^{q}\left(\mathbb{R}^{N}\right)$ be a distributional solution of

$$
-\Delta^{2} u=|u|^{q} \quad \text { on } \mathbb{R}^{N} .
$$

1. If $q(N-4) \leq N$, then $u \equiv 0$ a.e. in $\mathbb{R}^{N}$.

2. If $u \in L_{\text {loc }}^{N(q-1) / 4}\left(\mathbb{R}^{N}\right)$ and $1<q<\frac{N+4}{N-4}$, then $u \equiv 0$ a.e. in $\mathbb{R}^{N}$.

3. If $u$ is nontrivial, then $u<0$ a.e. and $\Delta u \geq 0$ in distributional sense.

4. If $q>\frac{N}{N-4}$, then (3.2) has nontrivial solutions (which is negative and subharmonic) in $\mathbb{R}^{N}$.

5. If $q \geq \frac{N+4}{N-4}$, then (3.2) has nontrivial smooth solutions (which is negative and subharmonic).

Notice that the above theorems imply that problems (3.1) and (3.2) do not admit nontrivial nonnegative solutions.

From Theorem 3.1 and Theorem 1.1 we can conjecture that if $u$ is a solution of

$$
-\Delta^{2} u \geq|u|^{q-1} u \text { on } \mathbb{R}^{N}
$$

then $u \leq 0$ a.e. in $\mathbb{R}^{N}$. However this conjecture is false as the following simple example shows. Let $u:=$ $1-x_{1}^{4} / 24$. The function $u$ changes sign, it is superharmonic and $-\Delta^{2} u=1$. Let $q>1$ and considering the function $f(t)=|t|^{q-1} t$. Since $1 \geq u$ and $f$ is increasing, we get that $-\Delta^{2} u=1 \geq|u|^{q-1} u$. This example shows that the conjecture is false even if we assume a sign on the Laplacian of the solution. Moreover, the above example also shows that a Kato inequality of the type

$$
u, f \in L_{l o c}^{1}(\Omega) \text { such that }-\Delta^{2} u \geq f \text {, then }-\Delta^{2} u^{+} \geq \operatorname{sign}^{+}(u) f,
$$

in distributional sense, cannot hold. Indeed if (3.4) holds, then $u^{+}$solves (3.1), and by Theorem 3.1 we obtain $u^{+} \equiv 0$, and this contradicts our counterexample.

Further remarks on the sign of the solutions of biharmonic inequalities and on the sign of their Laplacian will be considered in Section 7.2.

Proof of Theorem 3.1. Let $u$ be a distributional solution of (3.1). Multiplying by a test function $\phi_{R}$ as in (1.8), we have

$$
\begin{aligned}
& \int|u|^{q} \phi_{R} \leq-\int u \Delta^{2} \phi_{R} \leq \int|u|\left|\Delta^{2} \phi_{R}\right| \leq \int_{A_{R}}|u| \phi_{R}^{1 / q} \frac{\left|\Delta^{2} \phi_{R}\right|}{\phi_{R}^{1 / q}} \\
& \leq\left(\int_{A_{R}}|u|^{q} \phi_{R}\right)^{\frac{1}{q}}\left(\int_{A_{R}} \frac{\left|\Delta^{2} \phi_{R}\right|^{q^{\prime}}}{\phi_{R}^{q^{\prime}-1}}\right)^{\frac{1}{q^{\prime}}} \leq c_{1}\left(\int_{A_{R}}|u|^{q} \phi_{R}\right)^{\frac{1}{q}} R^{-4+\frac{N}{q^{\prime}}} .
\end{aligned}
$$

Therefore, we have

$$
\int_{B_{R}}|u|^{q} \leq c_{1} R^{-4 q^{\prime}+N},
$$

which in turn implies that

$$
f_{B_{R}}|u| \leq\left(f_{B_{R}}|u|^{q}\right)^{\frac{1}{q}} \leq c R^{-\frac{4}{q-1}}
$$

Proof of 1. If $N \leq 4$, from (3.6), by letting $R \rightarrow+\infty$ it follows that $u \equiv 0$ a.e. in $\mathbb{R}^{N}$. Let $N>4$ and $q \leq N /(N-4)$. From (3.6) by letting $R \rightarrow+\infty$, we deduce $\int_{\mathbb{R}^{N}}|u|^{q} \leq c_{1}<\infty$. This implies that

$$
\lim _{R} \int_{A_{R}}|u|^{q}=0,
$$


which plugged into (3.5) yields $|u|^{q} \equiv 0$ a.e. in $\mathbb{R}^{N}$.

Proof of 2. Let $N>4$ and let $u$ be a nontrivial solution of (3.1). By translation invariance from (3.7) we have,

$$
\liminf _{R \rightarrow+\infty} \frac{1}{R^{N}} \int_{R \leq|x-y| \leq 2 R}|u(y)| d y=0 \quad \text { for any } x \in \mathbb{R}^{N} .
$$

Hence, by Theorem A.1 applied to $v:=-u$, it follows that

$$
-u(x) \geq \frac{1}{C_{N}} \int \frac{|u(y)|^{q}}{|x-y|^{N-4}} d y, \quad \text { for any } x \in \mathbb{R}^{N},
$$

where $C_{N}$ is defined by (1.9). Clearly this implies that $-u$ is superharmonic in distributional sense in $\mathbb{R}^{N}$.

Proof of Theorem 3.2. Since (3.2) is a particular case of (3.1), statements 1. and 3. are a direct consequence of Theorem 3.1.

Arguing as in the proof of Theorem 3.1, it follows that the solutions of (3.2) can be represented by (3.9) with the equality sign. Therefore the function $v:=-u$ is a nonnegative superharmonic solution of

$$
\Delta^{2} v=v^{q} \quad \text { on } \mathbb{R}^{N},
$$

and of the integral equation

$$
v(x)=\frac{1}{C_{N}} \int \frac{v(y)^{q}}{|x-y|^{N-4}} d y, \text { for any } x \in \mathbb{R}^{N} .
$$

Proof of 2. Since $v \in L_{\text {loc }}^{N(q-1) / 4}\left(\mathbb{R}^{N}\right)$ and $1<q<\frac{N+4}{N-4}$, from Theorem 1.4 in [21], it follows that $v \equiv 0$ a.e. in $\mathbb{R}^{N}$. Proof of 4. Equation (3.10) admits singular solutions of the form $v(x)=c|x|^{-4 /(q-1)}$ for a suitable $c>0$.

Proof of 5. From [32] it follows that there exist infinitely many nontrivial radial positive smooth solutions of (3.10) (see also [18]), which yields our claim.

Similar results of those of Theorems 3.1 and Theorem $3.2(1 ., \cdots, 4$.) with the same proofs, can be proved for higher order problems of the type

$$
-(-\Delta)^{m} u \geq|u|^{q} \quad \text { on } \mathbb{R}^{N},
$$

and

$$
-(-\Delta)^{m} u=|u|^{q} \quad \text { on } \mathbb{R}^{N} .
$$

Let us to emphasize that for problems (3.12) and (3.13), the corresponding point 2. of Theorem 3.1 (and a fortiori, point 3. of Theorem 3.2) can be written as

$$
(-\Delta)^{i} u \leq 0 \text { in distributional sense, for } i=0, \ldots, m .
$$

The existence result for (3.13) for $m \geq 3$, like in 5. of Theorem 3.2, that is for $q>\frac{N+2 m}{N-2 m}$ is an open problem.

\section{On the notion of solution: other related problems}

In this paper we are mainly interested to the study possible solutions of the prototype equation

$$
-\Delta^{2} u=|u|^{q-1} u, \quad \text { on } \mathbb{R}^{N}
$$

which is, clearly a special case of the double inequality

$$
g(u) \geq-\Delta^{2} u \geq f(u), \quad \text { on } \mathbb{R}^{N}
$$


where $f, g: \mathbb{R} \rightarrow \mathbb{R}$ are given functions satisfying suitable assumptions. We emphasize that the methods that we are going to develop can be fruitfully used to study the solution of the one side inequality

$$
-u \Delta^{2} u \geq h \text { on } \mathbb{R}^{N} .
$$

We begin noticing that if $u \in \mathscr{C}^{4}\left(\mathbb{R}^{N}\right)$ is a solution of $\left({ }_{g} \mathbf{P}_{f}\right)$, then $u$ solves

$$
g(u) u^{+}-f(u) u^{-} \geq-u \Delta^{2} u \geq f(u) u^{+}-g(u) u^{-} \quad \text { on } \mathbb{R}^{N},
$$

where $u^{+}$and $u^{-}$are the positive and negative part of $u$ respectively. Indeed multiplying $\left(g \mathbf{P}_{f}\right)$ by $u^{+}$and $-u^{-}$, we have

$$
\begin{aligned}
& g(u) u^{+} \geq-\Delta^{2} u u^{+} \quad \geq f(u) u^{+}, \\
& -f(u) u^{-} \geq-\Delta^{2} u\left(-u^{-}\right) \geq-g(u) u^{-} .
\end{aligned}
$$

Summing these last two inequalities we obtain (4.1). Therefore, in what follow we shall study also possible solutions of the inequality $\left(\mathbf{P}_{h}\right)$.

Having in mind that in our main Liouville Theorems we are going to assume that

$$
f, g \in \mathscr{C}(\mathbb{R}), \quad f(t) t \geq 0, \quad g(t) t \geq 0, \quad \text { for any } t \in \mathbb{R},
$$

we see that $h=H(u)=f(u) u^{+}-g(u) u^{-} \geq 0$. However, it will be useful to study $\left(\mathbf{P}_{h}\right)$ without any assumption on the sign of $h \in L_{l o c}^{1}\left(\mathbb{R}^{N}\right)$. This extra generality, beside the fact that is interesting in itself, it will be essential when studying the distributional solutions of $\left({ }_{g} \mathbf{P}_{f}\right)$.

Definition 4.1. A function $u \in L_{l o c}^{1}\left(\mathbb{R}^{N}\right)$ is a distributional solution of $\left(g \mathbf{P}_{f}\right)$, if $f(u), g(u) \in L_{l o c}^{1}\left(\mathbb{R}^{N}\right)$ and

$$
\int g(u) \varphi \geq-\int u \Delta^{2} \varphi \geq \int f(u) \varphi,
$$

for any nonnegative $\varphi \in \mathscr{C}_{0}^{4}\left(\mathbb{R}^{N}\right)$.

A function $u \in H_{\text {loc }}^{2}\left(\mathbb{R}^{N}\right)$ is a weak solution of $\left(\mathbf{P}_{h}\right)$, if $h \in L_{\text {loc }}^{1}\left(\mathbb{R}^{N}\right)$ and

$$
\int-\Delta u \Delta(u \varphi) \geq \int h \varphi
$$

for any nonnegative $\varphi \in \mathscr{C}_{0}^{2}\left(\mathbb{R}^{N}\right)$.

Analogously, $u \in H_{\text {loc }}^{2}\left(\mathbb{R}^{N}\right)$ is a weak solution of (4.1), if $f(u) u, g(u) u \in L_{l o c}^{1}\left(\mathbb{R}^{N}\right)$ and

$$
\int\left(g(u) u^{+}-f(u) u^{-}\right) \varphi \geq-\int \Delta u \Delta(u \varphi) \geq \int\left(f(u) u^{+}-g(u) u^{-}\right) \varphi,
$$

for any nonnegative $\varphi \in \mathscr{C}_{0}^{2}\left(\mathbb{R}^{N}\right)$.

Theorem 4.2. Let $f, g \in \mathscr{C}\left(\mathbb{R}^{N}\right)$ and let $u \in H_{\text {loc }}^{2}\left(\mathbb{R}^{N}\right)$ be a distributional solution of $\left({ }_{g} \mathbf{P}_{f}\right)$ such that $u \in$ $L_{\text {loc }}^{s}\left(\mathbb{R}^{N}\right), 2 \leq s \leq \infty$ and $f(u), g(u) \in L_{\text {loc }}^{s^{\prime}}\left(\mathbb{R}^{N}\right)$. Then $u$ is a weak solution of (4.1).

Proof. Let $\left(m_{\eta}\right)_{\eta>0}$ be a family of standard mollifier cutoff functions. Let $u^{+}$and $u^{-}$be the positive and negative part of $u$ respectively. Then setting

$$
u_{\eta}:=u^{\star} m_{\eta}, \quad u_{\eta}^{+}:=\left(u^{+}\right)_{\eta}:=u^{+} \star m_{\eta}, \quad u_{\eta}^{-}:=\left(u^{-}\right)_{\eta}:=u^{-} \star m_{\eta},
$$

we have that $u_{\eta} \rightarrow u$ in $H_{l o c}^{2}\left(\mathbb{R}^{N}\right), u_{\eta} \rightarrow u, u_{\eta}^{+} \rightarrow u^{+}, u_{\eta}^{-} \rightarrow u^{-}$in $L_{l o c}^{s}\left(\mathbb{R}^{N}\right)$ and a.e. in $\mathbb{R}^{N}$.

Now using $\varphi u_{\eta}^{+}$and $\varphi u_{\eta}^{-}$as test functions in $\left.{ }_{g} \mathbf{P}_{f}\right)$ we get

$$
\int g(u) u_{\eta}^{+} \varphi \geq-\int \Delta u \Delta\left(u_{\eta}^{+} \varphi\right) \geq \int f(u) u_{\eta}^{+} \varphi,
$$




$$
\int g(u) u_{\eta}^{-} \varphi \geq-\int \Delta u \Delta\left(u_{\eta}^{-} \varphi\right) \geq \int f(u) u_{\eta}^{-} \varphi
$$

hence

$$
\int\left(g(u) u_{\eta}^{+}-f(u) u_{\eta}^{-}\right) \varphi \geq-\int \Delta u \Delta\left(\left(u_{\eta}^{+}-u_{\eta}^{-}\right) \varphi\right) \geq \int\left(f(u) u_{\eta}^{+}-g(u) u_{\eta}^{-}\right) \varphi .
$$

Since $u_{\eta}=u_{\eta}^{+}-u_{\eta}^{-} \rightarrow u$ in $H_{\text {loc }}^{2}\left(\mathbb{R}^{N}\right)$, we have $\int \Delta u \Delta\left(\left(u_{\eta}^{+}-u_{\eta}^{-}\right) \phi\right) \rightarrow \int \Delta u \Delta(u \phi)$. On the other hand, we know that $\int f(u) u_{\eta}^{+} \varphi \rightarrow \int f(u) u^{+} \varphi, \int f(u) u_{\eta}^{-} \varphi \rightarrow \int f(u) u^{-} \varphi, \int g(u) u_{\eta}^{+} \varphi \rightarrow \int g(u) u^{+} \varphi$ and $\int g(u) u_{\eta}^{-} \varphi \rightarrow \int g(u) u^{-} \varphi$, yielding

$$
\int\left(g(u) u^{+}-f(u) u^{-}\right) \varphi \geq-\int \Delta u \Delta(u \varphi) \geq \int\left(f(u) u^{+}-g(u) u^{-}\right) \varphi
$$

which is the claim.

Remark 4.3. From the above theorem we easily deduce that if $u \in H_{l o c}^{2}\left(\mathbb{R}^{N}\right)$ is a weak solution of $\left(g \mathbf{P}_{f}\right)$ with $f, g \in \mathscr{C}\left(\mathbb{R}^{N}\right)$, then $u$ is a weak solution of (4.1) provided one of the following conditions is satisfied

1. $u \in H_{\text {loc }}^{2}\left(\mathbb{R}^{N}\right) \cap L_{\text {loc }}^{q+1}\left(\mathbb{R}^{N}\right)$ and $C_{1} t^{q} \geq g(t) \geq f(t) \geq 0$ for $t \geq 0,0 \geq g(t) \geq f(t) \geq-C_{2}|t|^{q}$ for $t<0$, with suitable $C_{1}, C_{2}>0$

2. $g(u)=f(u)=|u|^{q-1} u, u \in H_{\text {loc }}^{2}\left(\mathbb{R}^{N}\right) \cap L_{\text {loc }}^{q+1}\left(\mathbb{R}^{N}\right)$;

3. $f(u) \in L_{\text {loc }}^{p_{1}}\left(\mathbb{R}^{N}\right), g(u) \in L_{\text {loc }}^{p_{2}}\left(\mathbb{R}^{N}\right)$ for some $p_{1}, p_{2} \geq \frac{2 N}{N+4}$;

4. $u \in H_{\text {loc }}^{2}\left(\mathbb{R}^{N}\right) \cap L_{\text {loc }}^{\infty}\left(\mathbb{R}^{N}\right)$.

Remark 4.4. When studying distributional solutions $u \in L_{l o c}^{1}\left(\mathbb{R}^{N}\right)$ of $\left(\mathrm{g} \mathbf{P}_{f}\right)$, we encounter several difficulties that can be overcome by analyzing the general problem $\left(\mathbf{P}_{h}\right)$ without the extra assumption on the sign of $h$.

\section{Asymptotic Hardy-Rellich type inequalities}

In order to develop the scheme described in Section 2 to our fourth order problem, we need to prove the counterpart of inequality (2.3). To this end an important step is to obtain some inequalities that we name Asymptotic Hardy-Rellich type inequalities. Let us point out why we call these inequalities asymptotic. It well known that for $u \in \mathscr{C}_{0}^{\infty}\left(\mathbb{R}^{N}\right), N>4$, the following inequalities

$$
\int|\Delta u|^{2} \geq \frac{N^{2}(N-4)^{2}}{16} \int|u|^{2} \frac{1}{|x|^{4}}, \quad \text { and } \int|\Delta u|^{2} \geq \frac{N^{2}}{4} \int|\nabla u|^{2} \frac{1}{|x|^{2}},
$$

holds (see for instance [30], [33]). Usually, in the literature the above inequalities hold for compactly supported functions $u$ and are known as Rellich type inequalities. If $u$ has not compact support and does not belong to some appropriate function space, say $D$, the above inequalities are not necessarily valid. However a version of these inequalities are satisfied by localization and by adding an error term, say $E_{1}(u)$. The latter may vanishes under suitable conditions.

The precise relation between the vanishing property of our error $E_{1}(u)$ and the fact that the function $u$ belongs to a suitable space $D$ is an interesting problem however we will not investigate this question in this paper.

Now we present some general inequalities that can be useful for further investigation. To the best of our knowledge the results in this section are new.

From the definition of weak solution (4.2) it is clear that we need to develop some estimates for integrals of the type

$$
\int \Delta u \Delta(u \varphi)
$$

To this end we observe that from Corollary 2.1 in [25] we deduce the following. 
Lemma 5.1. Let $\Omega \subset \mathbb{R}^{N}$ be an open set, $u \in \mathscr{C}^{2}(\Omega), H=\left(H^{1}, \ldots, H^{N}\right) \in \mathscr{C}^{1}\left(\Omega ; R^{N}\right)$ with $u$ or $H$ having compact support in $\Omega$. Then

$$
2 \int_{\Omega} \Delta u(H \cdot \nabla u)=\int_{\Omega} \operatorname{div}(H)|\nabla u|^{2}-2 \sum_{i, j=1}^{N} \int_{\Omega} \frac{\partial H^{j}}{\partial x_{i}} \frac{\partial u}{\partial x_{i}} \frac{\partial u}{\partial x_{j}} .
$$

Lemma 5.2. Let $\Omega \subset \mathbb{R}^{N}$ be an open set, $u, v \in \mathscr{C}^{2}(\Omega), \varphi \in \mathscr{C}^{1}(\Omega)$ with $v$ and $\varphi$ radial functions with at least one of them having compact support contained in $\Omega$. Then

$$
\begin{aligned}
& 2 \int_{\Omega} \Delta u(\nabla u \cdot \nabla v) \varphi=\int_{\Omega}|\nabla u|^{2} \Delta v \varphi+\int_{\Omega}|\nabla u|^{2} v^{\prime} \varphi^{\prime} \\
& -2 \int_{\Omega}\left\{\left(\nabla u \cdot \frac{x}{|x|}\right)^{2} v^{\prime} \varphi^{\prime}+\left(\nabla u \cdot \frac{x}{|x|}\right)^{2} v^{\prime \prime} \varphi-\left(\nabla u \cdot \frac{x}{|x|}\right)^{2} \frac{v^{\prime}}{|x|} \varphi+|\nabla u|^{2} \frac{v^{\prime}}{|x|} \varphi\right\} .
\end{aligned}
$$

Proof. Choose $H:=\varphi \nabla v$ in Lemma 5.1.

Our first main result is the following.

Theorem 5.3. Let $\Omega \subset \mathbb{R}^{N}$ be an open set. Let $v \in \mathscr{C}^{2}(\Omega)$ be a radial function such that $\frac{v^{\prime 2}}{|v|} \in L^{1}(\Omega)$. For any $u \in \mathscr{C}^{2}(\Omega)$, for any nonnegative radial $\varphi \in \mathscr{C}_{0}^{1}(\Omega)$, and $\delta>0$, we have

$$
\begin{aligned}
\int_{\Omega}|\Delta u|^{2}|v| \varphi \geq & \int_{\Omega}\left(\nabla u \cdot \frac{x}{|x|}\right)^{2} 2 \delta^{2}\left[v^{\prime \prime}-\frac{v^{\prime}}{|x|}-\frac{\delta^{2}}{2} \frac{v^{\prime 2}}{|v|}\right] \varphi \\
& +2 \delta^{2} \int_{\Omega}|\nabla u|^{2}\left[\frac{-\Delta v}{2}+\frac{v^{\prime}}{|x|}\right] \varphi+2 \delta^{2} E_{1}\left(\nabla u, v^{\prime}, \varphi^{\prime}\right),
\end{aligned}
$$

where

$$
E_{1}\left(\nabla u, v^{\prime}, \varphi^{\prime}\right):=\int_{\Omega}\left(\nabla u \cdot \frac{x}{|x|}\right)^{2} v^{\prime} \varphi^{\prime}-\frac{1}{2} \int_{\Omega}|\nabla u|^{2} v^{\prime} \varphi^{\prime} .
$$

In particular if $\varphi=\phi_{R}^{2}$, with $\phi_{R}$ defined in (1.8), and the estimate $\left|v^{\prime}\right| \leq c_{v} R^{\alpha-N-1}$ holds on $A_{R}$, then

$$
\left|E_{1}\left(\nabla u, v^{\prime}, \varphi^{\prime}\right)\right| \leq c_{v} c_{1}\left(\phi_{1}\right) R^{\alpha-2} f_{A_{R}}|\nabla u|^{2} \phi_{R} .
$$

Moreover, if in addition $-\Delta v+2 v^{\prime} /|x| \geq 0$, then

$$
\begin{aligned}
\int_{\Omega}|\Delta u|^{2}|v| \varphi \geq \int_{\Omega} & \left(\nabla u \cdot \frac{x}{|x|}\right)^{2} \delta^{2}\left[v^{\prime \prime}-(N-1) \frac{v^{\prime}}{|x|}-\delta^{2} \frac{v^{\prime 2}}{|v|}\right] \varphi \\
& +2 \delta^{2} E_{1}\left(\nabla u, v^{\prime}, \varphi^{\prime}\right) .
\end{aligned}
$$

Proof. By Cauchy-Schwarz inequality we have

$$
\begin{aligned}
\int_{\Omega} \Delta u(\nabla u \cdot \nabla v) \varphi & \leq\left(\int_{\Omega}|\Delta u|^{2} v \varphi\right)^{\frac{1}{2}}\left(\int_{\Omega}\left(\nabla u \cdot \frac{x}{|x|}\right)^{2} \frac{v^{\prime 2}}{v} \varphi\right)^{\frac{1}{2}} \\
& \leq \frac{1}{2 \delta^{2}} \int_{\Omega}|\Delta u|^{2} v \varphi+\frac{\delta^{2}}{2} \int_{\Omega}\left(\nabla u \cdot \frac{x}{|x|}\right)^{2} \frac{v^{\prime 2}}{v} \varphi,
\end{aligned}
$$

which plugged into (5.1) yields the inequality (5.2).

From the estimate

$$
\left|E_{1}\left(\nabla u, v^{\prime}, \varphi^{\prime}\right)\right| \leq \frac{3}{2} \int_{\Omega}|\nabla u|^{2}\left|v^{\prime}\right|\left|\varphi^{\prime}\right|=3 \int_{A_{R}}|\nabla u|^{2} \phi_{R}\left|v^{\prime}\right|\left|\phi_{R}^{\prime}\right|
$$


we deduce (5.4).

Finally, since $-\Delta v+2 \frac{v^{\prime}}{|x|} \geq 0$, the inequality

$$
|\nabla u|^{2} \geq\left(\nabla u \cdot \frac{x}{|x|}\right)^{2}
$$

combined with (5.2) yields inequality (5.5).

For compactly supported function we have the following

Corollary 5.4. Let $v \in \mathscr{C}^{2}\left(\mathbb{R}^{N}\right)$ be a radial function such that $\frac{v^{\prime 2}}{|v|} \in L_{\text {loc }}^{1}\left(\mathbb{R}^{N}\right)$. For any $u \in \mathscr{C}_{0}^{2}\left(\mathbb{R}^{N}\right)$, and $\delta>0$, we have

$$
\begin{array}{r}
\int_{\mathbb{R}^{N}}|\Delta u|^{2}|v| \geq \int_{\mathbb{R}^{N}}\left(\nabla u \cdot \frac{x}{|x|}\right)^{2} 2 \delta^{2}\left[v^{\prime \prime}-\frac{v^{\prime}}{|x|}-\frac{\delta^{2}}{2} \frac{v^{\prime 2}}{|v|}\right] \\
+2 \delta^{2} \int_{\mathbb{R}^{N}}|\nabla u|^{2}\left[\frac{-\Delta v}{2}+\frac{v^{\prime}}{|x|}\right] .
\end{array}
$$

Moreover, if in addition $-\Delta v+2 v^{\prime} /|x| \geq 0$, then

$$
\int_{\mathbb{R}^{N}}|\Delta u|^{2}|v| \geq \int_{\mathbb{R}^{N}}\left(\nabla u \cdot \frac{x}{|x|}\right)^{2} \delta^{2}\left[v^{\prime \prime}-(N-1) \frac{v^{\prime}}{|x|}-\delta^{2} \frac{v^{\prime 2}}{|v|}\right] .
$$

Proof. Let $u \in \mathscr{C}_{0}^{2}\left(\mathbb{R}^{N}\right)$. We choose $\varphi=\phi_{R}$ with $R$ large enough such that the support of $u$ is contained in the ball of radius $R$. With this choice of $\phi$, the term $E_{1}$ which appears in (5.2) and (5.5) vanishes. Taking into account that $\varphi=1$ on the support of $u$, the claim follows.

Remark 5.5. Since in Theorem 5.3 and Corollary 5.4 there is no assumption on the sign of $v$, we notice that if

$$
-\Delta v+2 \frac{v^{\prime}}{|x|} \leq 0
$$

then (5.5) and (5.7) still hold replacing $v$ by $-v$ (as well as its derivatives).

In what follows we deal with a particular weight $v_{\epsilon}$ that we are going to define below. From now on we set

$$
r:=|x|, \text { and for } \epsilon>0, r_{\epsilon}:=\left(\epsilon^{2}+|x|^{2}\right)^{1 / 2}=\left(\epsilon^{2}+r^{2}\right)^{1 / 2},
$$

and define

$$
v_{\epsilon}(x)=v_{\epsilon}(|x|)=v_{\epsilon}(r):=\frac{1}{\left(\epsilon^{2}+r^{2}\right)^{\frac{N-\alpha}{2}}}=r_{\epsilon}^{\alpha-N},
$$

where $\alpha \in \mathbb{R}$. A simple computation gives

$$
\begin{gathered}
-\Delta v_{\epsilon}=-\Delta r_{\epsilon}^{\alpha-N}=(N-\alpha) \frac{(\alpha-2) r^{2}+N \epsilon^{2}}{\left(\epsilon^{2}+r^{2}\right)^{\frac{N-\alpha+4}{2}}}, \\
\Delta^{2} v_{\epsilon}=\Delta^{2} r_{\epsilon}^{\alpha-N}=(N-\alpha)(N+2-\alpha) \times \\
\times \frac{(\alpha-2)(\alpha-4) r^{4}+2(\alpha-4)(N+2) \epsilon^{2} r^{2}+N(N+2) \epsilon^{4}}{\left(\epsilon^{2}+r^{2}\right)^{\frac{N-\alpha+8}{2}}}, \\
\left|\nabla v_{\epsilon}\right|=\frac{|N-\alpha| r}{\left(\epsilon^{2}+r^{2}\right)^{\frac{N-\alpha+2}{2}}} .
\end{gathered}
$$

Therefore, by choosing $N>\alpha \geq 4$, it follows that $v_{\epsilon}$ is a positive super-biharmonic function namely, $-\Delta v_{\epsilon}>0$, $\Delta^{2} v_{\epsilon}>0$. Furthermore on $A_{R}=B_{2 R} \backslash B_{R}$ the following estimates hold

$$
v_{\epsilon}(x) \leq|x|^{\alpha-N}, \quad\left|\nabla v_{\epsilon}(x)\right| \leq|N-\alpha||x|^{\alpha-1-N},
$$




$$
\left|\Delta v_{\epsilon}(x)\right| \leq c|x|^{\alpha-2-N}, \quad\left|\nabla \Delta v_{\epsilon}(x)\right| \leq c|x|^{\alpha-3-N},
$$

where $c=c(N, \alpha)>0$ is a suitable positive constant independent of $\epsilon$.

As particular case of Theorem 5.3 we have the following.

Theorem 5.6. Let $N \geq 1$ and $\alpha \in \mathbb{R}$. For any $u \in H_{\text {loc }}^{2}\left(\mathbb{R}^{N}\right), t \in \mathbb{R}, \epsilon>0$, and a radial nonnegative function $\varphi \in \mathscr{C}_{0}^{1}\left(\mathbb{R}^{N}\right)$, we have

$$
\begin{aligned}
\int_{\mathbb{R}^{N}}|\Delta u|^{2} \frac{\varphi}{r_{\epsilon}^{N-\alpha}} \geq t(2 N & -2 \alpha+4-t) \int_{\mathbb{R}^{N}}\left(\nabla u \cdot \frac{x}{|x|}\right)^{2} \varphi \frac{r^{2}}{r_{\epsilon}^{N-\alpha+4}} \\
+ & t(\alpha-4) \int_{\mathbb{R}^{N}}|\nabla u|^{2} \varphi \frac{r^{2}}{r_{\epsilon}^{N-\alpha+4}} \\
& +t(N-2) \epsilon^{2} \int_{\mathbb{R}^{N}}|\nabla u|^{2} \varphi \frac{1}{r_{\epsilon}^{N-\alpha+4}} \\
& -2 t E_{1}\left(\nabla u, \frac{r}{r_{\epsilon}^{N-\alpha+2}}, \varphi^{\prime}\right) .
\end{aligned}
$$

In particular if $2 N>\alpha \geq 4$ or $N=1$ and $4>\alpha>2$, we have

$$
\begin{gathered}
\int_{\mathbb{R}^{N}}|\Delta u|^{2} \frac{\varphi}{r_{\epsilon}^{N-\alpha}} \geq\left(\frac{2 N-\alpha}{2}\right)^{2} \int_{\mathbb{R}^{N}}\left(\nabla u \cdot \frac{x}{|x|}\right)^{2} \varphi \frac{r^{2}}{r_{\epsilon}^{N-\alpha+4}} \\
-(2 N-\alpha) E_{1}\left(\nabla u, \frac{r}{r_{\epsilon}^{N-\alpha+2}}, \varphi^{\prime}\right), \\
\int_{\mathbb{R}^{N}}|\Delta u|^{2} \frac{\varphi}{r_{\epsilon}^{N-\alpha}} \geq H_{\alpha} \int_{\mathbb{R}^{N}}|\nabla u|^{2} \varphi \frac{r^{2}}{r_{\epsilon}^{N-\alpha+4}}-2 t_{\alpha} E_{1}\left(\nabla u, \frac{r}{r_{\epsilon}^{N-\alpha+2}}, \varphi^{\prime}\right),
\end{gathered}
$$

where

$$
\begin{gathered}
\text { if } 2 N>\alpha \geq \frac{2 N+8}{3}, \text { then } \begin{array}{l}
t_{\alpha}:=\frac{2 N-\alpha}{2}, \\
H_{\alpha}:=t_{\alpha}\left(2 N-\alpha-t_{\alpha}\right)=\left(\frac{2 N-\alpha}{2}\right)^{2},
\end{array} \\
\text { if } \frac{2 N+8}{3}>\alpha>4 \text {, then } \begin{array}{l}
t_{\alpha}:=2(N+2-\alpha), \\
H_{\alpha}:=t_{\alpha}\left(2 N-\alpha-t_{\alpha}\right)=2(N+2-\alpha)(\alpha-4) .
\end{array}
\end{gathered}
$$

Moreover, if $\varphi=\phi_{R}^{2}$ then

$$
\left|E_{1}\left(\nabla u, \frac{r}{r_{\epsilon}^{N-\alpha+2}}, \varphi^{\prime}\right)\right| \leq\left|E_{1}\left(\nabla u, \frac{1}{|x|^{N-\alpha+1}}, \varphi^{\prime}\right)\right| \leq c_{1}\left(\phi_{1}\right) R^{\alpha-2} f_{A_{R}}|\nabla u|^{2} \phi_{R} .
$$

A version of the above inequalities for singular weight is contained in the following.

Theorem 5.7. Let $N \geq 1$ and $\alpha \in \mathbb{R}$. Let $u \in \mathscr{C}^{2}\left(\mathbb{R}^{N}\right), t \in \mathbb{R}$, and let $\varphi \in \mathscr{C}_{0}^{1}\left(\mathbb{R}^{N}\right)$ be a radial nonnegative function. If one of the following cases holds

1. $u \equiv 0$ or $\varphi \equiv 0$ in a neighborhood of $0, N \geq 1, \alpha \in \mathbb{R}, t \in \mathbb{R}$,

2. $N \geq 2, \alpha>2, t \geq 0$,

then we have

$$
\begin{aligned}
\int_{\mathbb{R}^{N}}|\Delta u|^{2} \frac{\varphi}{|x|^{N-\alpha}} \geq & t(2 N-2 \alpha+4-t) \int_{\mathbb{R}^{N}}\left(\nabla u \cdot \frac{x}{|x|}\right)^{2} \frac{\varphi}{|x|^{N-\alpha+2}} \\
& +t(\alpha-4) \int_{\mathbb{R}^{N}}|\nabla u|^{2} \frac{\varphi}{|x|^{N-\alpha+2}}-2 t E_{1}\left(\nabla u, \frac{1}{|x|^{N-\alpha+1}}, \varphi^{\prime}\right) .
\end{aligned}
$$


In particular if $2 N>\alpha \geq 4$, then

$$
\begin{gathered}
\int_{\mathbb{R}^{N}}|\Delta u|^{2} \frac{\varphi}{|x|^{N-\alpha}} \geq\left(\frac{2 N-\alpha}{2}\right)^{2} \int_{\mathbb{R}^{N}}\left(\nabla u \cdot \frac{x}{|x|}\right)^{2} \frac{\varphi}{|x|^{N-\alpha+2}} \\
-(2 N-\alpha) E_{1}\left(\nabla u, \frac{1}{|x|^{N-\alpha+1}}, \varphi^{\prime}\right), \\
\int_{\mathbb{R}^{N}}|\Delta u|^{2} \frac{\varphi}{|x|^{N-\alpha}} \geq H_{\alpha} \int_{\mathbb{R}^{N}}|\nabla u|^{2} \varphi \frac{1}{|x|^{N-\alpha+2}}-2 t_{\alpha} E_{1}\left(\nabla u, \frac{1}{|x|^{N-\alpha+1}}, \varphi^{\prime}\right),
\end{gathered}
$$

where $H_{\alpha}$ and $t_{\alpha}$ are defined in (5.14) and (5.15).

Moreover, if $\varphi=\phi_{R}^{2}$ then (5.16) holds.

For radial functions we have the following.

Corollary 5.8. Let $2 N>\alpha>2$. For any radial $u \in \mathscr{C}^{2}\left(\mathbb{R}^{N}\right)$, the inequality (5.18) holds.

Proof. Since $\left(\nabla u \cdot \frac{x}{|x|}\right)^{2}=|\nabla u|^{2}$, and $N>2$ from (5.17), for $t>0$, we have

$$
\int|\Delta u|^{2} \frac{\varphi}{|x|^{N-\alpha}} \geq t(2 N-\alpha-t) \int|\nabla u|^{2} \frac{\varphi}{|x|^{N-\alpha+2}}-2 t E_{1}\left(\nabla u, \frac{1}{|x|^{N-\alpha+1}}, \varphi^{\prime}\right) .
$$

The conclusion follows by choosing $t=(2 N-\alpha) / 2$.

Dealing with compactly supported functions we have the following.

Corollary 5.9. Let $N \geq 2, \alpha>2, t \geq 0$. For any $u \in \mathscr{C}_{0}^{2}\left(\mathbb{R}^{N}\right)$, we have

$$
\begin{array}{r}
\int_{\mathbb{R}^{N}}|\Delta u|^{2} \frac{1}{|x|^{N-\alpha}} \geq t(2 N-2 \alpha+4-t) \int_{\mathbb{R}^{N}}\left(\nabla u \cdot \frac{x}{|x|}\right)^{2} \frac{1}{|x|^{N-\alpha+2}} \\
+t(\alpha-4) \int_{\mathbb{R}^{N}}|\nabla u|^{2} \frac{1}{|x|^{N-\alpha+2}}
\end{array}
$$

In particular if $2 N>\alpha \geq 4$, then

$$
\begin{aligned}
& \int_{\mathbb{R}^{N}}|\Delta u|^{2} \frac{1}{|x|^{N-\alpha}} \geq\left(\frac{2 N-\alpha}{2}\right)^{2} \int_{\mathbb{R}^{N}}\left(\nabla u \cdot \frac{x}{|x|}\right)^{2} \frac{1}{|x|^{N-\alpha+2}}, \\
& \int_{\mathbb{R}^{N}}|\Delta u|^{2} \frac{1}{|x|^{N-\alpha}} \geq H_{\alpha} \int_{\mathbb{R}^{N}}|\nabla u|^{2} \frac{1}{|x|^{N-\alpha+2}},
\end{aligned}
$$

where $H_{\alpha}$ is defined in (5.14) and (5.15).

If $2 N>\alpha>2$ and $u \in \mathscr{C}_{0}^{2}\left(\mathbb{R}^{N}\right)$ is radial, then (5.21) holds.

The proof is similar to the proof of Corollary 5.4.

Remark 5.10. Observe that if $2 N>\alpha \geq(2 N+8) / 3$, then $H_{\alpha}=(2 N-\alpha)^{2} / 4$ and (5.13) $\Longrightarrow(5.12),(5.19) \Longrightarrow(5.18)$, and (5.22) $\Longrightarrow$ (5.21).

Remark 5.11. In [33, Theorems 1.7 and 6.4] the authors prove that the inequality

$$
\int_{\mathbb{R}^{N}}|\Delta u|^{2} \frac{1}{|x|^{N-\alpha}} \geq B_{\alpha} \int_{\mathbb{R}^{N}}|\nabla u|^{2} \frac{1}{|x|^{N-\alpha+2}}, \quad \forall u \in \mathscr{C}_{0}^{\infty}\left(\mathbb{R}^{N}\right),
$$


holds for $N \geq \alpha>4$, where the best constant $B_{\alpha}$ is given by $B_{\alpha}=\left(\frac{2 N-\alpha}{2}\right)^{2}$ whenever $\frac{4(N+1)-2 \sqrt{N^{2}-N+1}}{3} \leq \alpha \leq N$, while $0<B_{\alpha}<\left(\frac{2 N-\alpha}{2}\right)^{2}$ for $4<\alpha<\frac{4(N+1)-2 \sqrt{N^{2}-N+1}}{3}$.

From (5.21), and the fact that $|\nabla u|^{2} \geq\left(\nabla u \cdot \frac{x}{|x|}\right)$, we deduce that for $4<\alpha<\frac{4(N+1)-2 \sqrt{N^{2}-N+1}}{3}$, the minimizing sequence related to (5.23) is not radial.

We observe that the range $2 N>\alpha>N$ has not been considered in [33]. However, inequalities (5.21) and (5.22) are still valid in that range.

It seems an interesting problem to study the sharpness of the constant appearing in (5.21).

Proof of Theorems 5.6 and 5.7. We begin proving the results for $u \in \mathscr{C}^{2}\left(\mathbb{R}^{N}\right)$ and for $\alpha \neq N$. The results follows from Theorem 5.3 by choosing $v=\gamma v_{\epsilon}$ where $v_{\epsilon}$ is defined in (5.9) and $\gamma$ can assume only two values, $\gamma=1$ or $\gamma=-1$. With this choice, we have

$$
\begin{aligned}
& v^{\prime \prime}-\frac{v^{\prime}}{r}-\frac{\delta^{2}}{2} \frac{v^{\prime 2}}{|v|}=(N-\alpha) \gamma\left(N-\alpha+2-\frac{\delta^{2}}{2} \gamma(N-\alpha)\right) \frac{r^{2}}{r_{\epsilon}^{N-\alpha+4}}, \\
& \frac{-\Delta v}{2}+\frac{v^{\prime}}{r}=\frac{(N-\alpha) \gamma(\alpha-4)}{2} \frac{r^{2}}{r_{\epsilon}^{N-\alpha+4}}+\epsilon^{2} \frac{(N-\alpha) \gamma(N-2)}{2} \frac{1}{r_{\epsilon}^{N-\alpha+4}} .
\end{aligned}
$$

Plugging these quantity in (5.2), setting $t:=\delta^{2}(N-\alpha) \gamma$ we deduce (5.11) for any $t \neq 0$ (since $\delta^{2}$ runs on all positive numbers and $\gamma$ can be choose in $\{-1,1\})$. The case $t=0$ is trivial. The case $\alpha=N$ follows by letting $\alpha \rightarrow N$ in (5.11).

With the choice $t=\frac{2 N-\alpha}{2}$ in (5.11), since $t(\alpha-4) \geq 0, t(N-2) \geq 0$ and taking into account that

$$
|\nabla u|^{2} \geq\left(\nabla u \cdot \frac{x}{|x|}\right)^{2},
$$

we deduce (5.12).

In order to prove (5.13), we use (5.11) by choosing $t=t_{\alpha}$ with $t_{\alpha}$ as defined in (5.14) or in (5.15). With this choice, the coefficient of the integral involving $\left(\nabla u \cdot \frac{x}{|x|}\right)^{2}$ is nonpositive, $t_{\alpha}\left(2 N-2 \alpha+4-t_{\alpha}\right) \leq 0$, and by (5.24) we obtain the claim.

The inequalities in Theorem 5.7 follow by letting $\epsilon \rightarrow 0$ in (5.11), (5.12) and (5.13). To this end, we notice that in the case $\alpha=4$ the term containing the weight $r_{\epsilon}^{-N+\alpha-4}$ is too singular when $\epsilon \rightarrow 0$, however taking into account that it is nonnegative, it can be ignored.

Finally, the estimate (5.16), easily follows from the definition of $E_{1}$.

The case $u \in H_{\text {loc }}^{2}\left(\mathbb{R}^{N}\right)$ in Theorem 5.6 follows by a regularization argument.

\subsection{An integral identity}

Lemma 5.12. Let $u \in H_{\text {loc }}^{2}\left(\mathbb{R}^{N}\right), \varphi \in \mathscr{C}_{0}^{4}\left(\mathbb{R}^{N}\right)$ and $v \in \mathscr{C}^{4}\left(\mathbb{R}^{N}\right)$. We have

$$
\begin{aligned}
\int \Delta u \Delta(u v \varphi) & =\int(\Delta u)^{2} v \varphi+\frac{1}{2} \int u^{2} \Delta^{2} v \varphi-\int|\nabla u|^{2} \Delta v \varphi \\
+ & 2 \int \Delta u \varphi(\nabla v, \nabla u)+\frac{1}{2} \int u^{2} \Delta v \Delta \varphi+\int u^{2}(\nabla \Delta v, \nabla \varphi) \\
+ & \int \Delta u u v \Delta \varphi+2 \int \Delta u u(\nabla v, \nabla \varphi)+2 \int \Delta u v(\nabla u, \nabla \varphi) .
\end{aligned}
$$

Furthermore if $\varphi$ and $v$ are assumed to be radial, then

$$
\begin{aligned}
& \int \Delta u \Delta(u v \varphi)=\int(\Delta u)^{2} v \varphi+\frac{1}{2} \int u^{2} \Delta^{2} v \varphi-2 \int\left(\nabla u \cdot \frac{x}{|x|}\right)^{2} v^{\prime \prime} \varphi \\
& -2 \int|\nabla u|^{2} \frac{v^{\prime}}{|x|} \varphi+2 \int\left(\nabla u \cdot \frac{x}{|x|}\right)^{2} \frac{v^{\prime}}{|x|} \varphi-2 E_{1}\left(\nabla u, v^{\prime}, \varphi^{\prime}\right)+E_{2}(u, v, \varphi)
\end{aligned}
$$


where $E_{1}$ is defined in (5.3) and $E_{2}$ is defined as

$$
\begin{aligned}
E_{2}(u, v, \varphi):=\int & u^{2} \Delta v \Delta \varphi+\int u^{2}(\nabla \Delta v, \nabla \varphi)+\int \Delta u u v \Delta \varphi \\
& +2 \int \Delta u u(\nabla v \cdot \nabla \varphi)+2 \int \Delta u v(\nabla u \cdot \nabla \varphi) .
\end{aligned}
$$

In particular if $\varphi=\phi_{R}^{4}$, with $\phi_{R}$ defined in (1.8), and

$$
\text { for } k=0,1,2,3, \quad\left|v^{(k)}\right| \leq c_{v} R^{\alpha-N-k}
$$

holds on $A_{R}$, then

$$
\begin{aligned}
& \left|E_{2}(u, v, \varphi)\right| \leq c_{v} c_{1}\left(\phi_{1}\right) R^{\alpha} \times \\
& \times\left[R^{-4} f_{A_{R}}|u|^{2} \phi_{R}+\left(f_{A_{R}}|\Delta u|^{2} \phi_{R}\right)^{\frac{1}{2}}\left(R^{-4} f_{A_{R}}|u|^{2} \phi_{R}\right)^{\frac{1}{2}}\right. \\
& \left.\quad+\left(f_{A_{R}}|\Delta u|^{2} \phi_{R}\right)^{\frac{1}{2}}\left(R^{-2} f_{A_{R}}\left(\nabla u \cdot \frac{x}{|x|}\right)^{2} \phi_{R}\right)^{\frac{1}{2}}\right] .
\end{aligned}
$$

Proof. We prove the claim for $u \in \mathscr{C}^{2}\left(\mathbb{R}^{N}\right)$. The general case follows by a regularization argument. Let $\varphi \in$ $\mathscr{C}_{0}^{4}\left(\mathbb{R}^{N}\right)$ and $v \in \mathscr{C}^{4}\left(\mathbb{R}^{N}\right)$. We have

$$
\begin{aligned}
& \int \Delta u \Delta(u v \varphi) \\
& =\int(\Delta u)^{2} v \varphi+\int \Delta u u \Delta v \varphi+\int \Delta u u v \Delta \varphi+ \\
& \quad+2 \int \Delta u u(\nabla v, \nabla \varphi)+2 \int \Delta u v(\nabla u, \nabla \varphi)+2 \int \Delta u \varphi(\nabla v, \nabla u) .
\end{aligned}
$$

By using the identity

$$
\Delta u^{2}=2 u \Delta u+2|\nabla u|^{2},
$$

the second term in right hand side of (5.30) can be rewritten as

$$
\begin{array}{rl}
\int \Delta u & u \Delta v=\frac{1}{2} \int \Delta\left(u^{2}\right) \Delta v \varphi-\int|\nabla u|^{2} \Delta v \varphi \\
= & \frac{1}{2} \int u^{2} \Delta(\Delta v \varphi)-\int|\nabla u|^{2} \Delta v \varphi \\
= & \frac{1}{2} \int u^{2} \Delta^{2} v \varphi+\frac{1}{2} \int u^{2} \Delta v \Delta \varphi+\int u^{2}(\nabla \Delta v, \nabla \varphi)-\int|\nabla u|^{2} \Delta v \varphi .
\end{array}
$$

Hence, (5.30) can be rewritten as in the claim.

Taking into account the identity in Lemma 5.1, from (5.25) we get (5.26).

The estimate (5.29) can be proved by Cauchy-Schwarz inequality and by using the estimates (5.28) and the estimates on the derivatives of $\phi_{R}$. For instance, since

$$
\left|\Delta \phi_{R}^{4}\right|=\left.\left|4 \phi_{R}^{3} \Delta \phi_{R}+12 \phi_{R}^{2}\right| \nabla \phi_{R}\right|^{2} \mid \leq c_{1}\left(\phi_{1}\right) \phi_{R} R^{-2}
$$

(we have used the fact that $\phi_{R}^{k} \leq \phi_{R}$ for any $k \geq 1$ ), we have

$$
\begin{aligned}
\left|\int \Delta u u v \Delta \phi_{R}^{4}\right| & \leq c_{1} \int|\Delta u| \phi_{R} R^{-2}|u| c_{v} R^{\alpha-N} \\
& \leq c_{1} c_{v}\left(R^{\alpha} f_{A_{R}}|\Delta u|^{2} \phi_{R}\right)^{\frac{1}{2}}\left(R^{\alpha-4} f_{A_{R}}|u|^{2} \phi_{R}\right)^{\frac{1}{2}} .
\end{aligned}
$$

The other terms can be estimated in a similar way.

Choosing $v=v_{\epsilon}$ in the above Lemma and arguing as in the proof of Proposition 5.6, it follows that 
Proposition 5.13. Let $N>4, \alpha \geq 4$, and $u \in \mathscr{C}^{4}\left(\mathbb{R}^{N}\right)$. For any radial $\varphi \in \mathscr{C}_{0}^{4}\left(\mathbb{R}^{N}\right)$, we have

$$
\begin{aligned}
\int \Delta u \Delta\left(u \frac{\varphi}{|x|^{N-\alpha}}\right)= & \int(\Delta u)^{2} \frac{\varphi}{|x|^{N-\alpha}}+2(N-\alpha) \int|\nabla u|^{2} \frac{\varphi}{|x|^{N-\alpha+2}} \\
& -2(N-\alpha)(N+2-\alpha) \int\left(\nabla u \cdot \frac{x}{|x|}\right)^{2} \frac{\varphi}{|x|^{N-\alpha+2}} \\
& +(N-\alpha)(N+2-\alpha) P\left(u^{2} \varphi, \alpha\right) \\
& +2(N-\alpha) E_{1}\left(\nabla u, \frac{1}{|x|^{N-\alpha+1}}, \varphi^{\prime}\right)+E_{2}\left(u,|x|^{\alpha-N}, \varphi\right)
\end{aligned}
$$

where $E_{1}$ and $E_{2}$ are defined respectively in (5.3) and (5.32) and $P$ is defined as

$$
P\left(u^{2} \varphi, \alpha\right):= \begin{cases}\frac{(\alpha-2)(\alpha-4)}{2} \int u^{2} \frac{\varphi}{|x|^{N-\alpha+4},} & \text { if } \alpha>4, \\ \frac{C_{N}}{2(N-4)(N-2)} u^{2}(0) \varphi(0), & \text { if } \alpha=4,\end{cases}
$$

where $C_{N}>0$ is the positive constant defined in (1.9).

\subsection{Some functional weighted quadratic inequalities}

Gluing the identity (5.26) and the inequality (5.2) we deduce an inequality for the bilinear form

$$
u \in \mathscr{C}^{2}\left(\mathbb{R}^{N}\right) \longmapsto \int \Delta u \Delta(u v \varphi)
$$

for a general radial nonnegative weight $v \in \mathscr{C}^{4}\left(\mathbb{R}^{N}\right)$.

Theorem 5.14. Let $u \in \mathscr{C}^{2}\left(\mathbb{R}^{N}\right)$. Let $\varphi \in \mathscr{C}_{0}^{4}\left(\mathbb{R}^{N}\right), v \in \mathscr{C}^{4}\left(\mathbb{R}^{N}\right)$ be radial nonnegative functions and $\delta>0$ be such that

$$
\delta^{2}\left(-\Delta v+2 \frac{v^{\prime}}{|x|}\right)-2 \frac{v^{\prime}}{|x|} \geq 0, \quad \frac{v^{\prime 2}}{v} \in L_{l o c}^{1}\left(\mathbb{R}^{N}\right) .
$$

Then

$$
\begin{aligned}
\int \Delta u \Delta(u v \varphi) \geq & \frac{1}{2} \int u^{2} \Delta^{2} v \varphi \\
& +\int\left(\nabla u \cdot \frac{x}{|x|}\right)^{2}\left[2 \delta^{2}\left(v^{\prime \prime}-\frac{1}{2} \Delta v-\frac{\delta^{2}}{2} \frac{v^{\prime 2}}{v}\right)-2 v^{\prime \prime}\right] \varphi \\
& +2\left(\delta^{2}-1\right) E_{1}\left(\nabla u, v^{\prime}, \varphi^{\prime}\right)+E_{2}(u, v, \varphi) .
\end{aligned}
$$

Proof. Gluing the identity (5.26) and the inequality (5.2) we obtain

$$
\begin{aligned}
\int \Delta u \Delta(u v \varphi) \geq & \frac{1}{2} \int u^{2} \Delta^{2} v \varphi \\
& +\int\left(\nabla u \cdot \frac{x}{|x|}\right)^{2}\left[2 \delta^{2}\left(v^{\prime \prime}-\frac{v^{\prime}}{|x|}-\frac{\delta^{2}}{2} \frac{v^{\prime 2}}{v}\right)-2 v^{\prime \prime}+2 \frac{v^{\prime}}{|x|}\right] \varphi \\
& +\int|\nabla u|^{2}\left[2 \delta^{2}\left(-\frac{1}{2} \Delta v+\frac{v^{\prime}}{|x|}\right)-2 \frac{v^{\prime}}{|x|}\right] \varphi \\
& +2\left(\delta^{2}-1\right) E_{1}\left(\nabla u, v^{\prime}, \varphi^{\prime}\right)+E_{2}(u, v, \varphi) .
\end{aligned}
$$

By using the hypothesis (5.33) together with (5.24), we get the claim. 
Theorem 5.15. Let $N>4$ and $N+\sqrt{N^{2}-4 N} \geq \alpha \geq N-\sqrt{N^{2}-4 N}$. Let $u \in H_{\text {loc }}^{2}\left(\mathbb{R}^{N}\right)$ and let $r$, $r_{\epsilon}$ be as in (5.8). For any $\varphi \in \mathscr{C}_{0}^{4}\left(\mathbb{R}^{N}\right)$, radial and nonnegative functions, we have

$$
\begin{aligned}
\int \Delta u \Delta(u & \left.\frac{\varphi}{r_{\epsilon}^{N-\alpha}}\right) \geq \frac{1}{2} \int u^{2} \Delta^{2}\left(r_{\epsilon}^{\alpha-N}\right) \varphi \\
& +\frac{C(N, \alpha)}{4} \int_{\mathbb{R}^{N}}\left(\nabla u \cdot \frac{x}{|x|}\right)^{2} \frac{r^{2}}{r_{\epsilon}^{N-\alpha+4}} \varphi \\
& +\frac{\epsilon^{2}}{2}((2 N-\alpha)(N-2)+4(N-\alpha)) \int_{\mathbb{R}^{N}}|\nabla u|^{2} \frac{1}{r_{\epsilon}^{N-\alpha+4}} \varphi \\
& -\alpha E_{1}\left(\nabla u, \frac{r}{r_{\epsilon}^{N-\alpha+2}}, \varphi^{\prime}\right)+E_{2}\left(u, r_{\epsilon}^{\alpha-N}, \varphi\right)
\end{aligned}
$$

where

$$
C(N, \alpha):=-7 \alpha^{2}+4(2+3 N) \alpha-4 N(N+2),
$$

and $E_{1}$, and $E_{2}$ are defined in (5.3) and (5.27) respectively.

Remark 5.16. Let us analyze the positivity of some terms appearing in (5.35).

i) Let

$$
\begin{aligned}
& \alpha_{1}:=\frac{2}{7}\left(3 N+2-\sqrt{2\left(N^{2}-N+2\right)}\right), \\
& \alpha_{2}:=\frac{2}{7}\left(3 N+2+\sqrt{2\left(N^{2}-N+2\right)}\right),
\end{aligned}
$$

be the two roots of $C(N, \alpha)$. Clearly, the constant $C(N, \alpha)$ is non negative for $\alpha_{1} \leq \alpha \leq \alpha_{2}$.

ii) The coefficient $\frac{\epsilon^{2}}{2}((2 N-\alpha)(N-2)+4(N-\alpha))$ is nonnegative for $\alpha \leq \frac{2 N^{2}}{N+2}$.

iii) From (5.10), we have $\Delta^{2} r_{\epsilon}^{\alpha-N}$ is nonnegative provided $\alpha \geq 4$ and $(N-\alpha)(N+2-\alpha) \geq 0$.

iv) For $N>4$, we have

$$
N-\sqrt{N^{2}-4 N}<\alpha_{1}<N<\alpha_{2}<\frac{2 N^{2}}{N+2}<N+\sqrt{N^{2}-4 N},
$$

with $4<\alpha_{1}$ for $N \geq 8$ and $\alpha_{1}<4$ for $N=5,6,7$.

Therefore for $N \geq \alpha \geq \max \left\{4, \alpha_{1}\right\}$, it follows $C(N, \alpha)$ is nonnegative as well as the coefficient $\frac{\epsilon^{2}}{2}((2 N-\alpha)(N-$ $2)+4(N-\alpha)$ ) and the integral involving $\Delta^{2} r_{\epsilon}^{\alpha-N}$. This remark allow us to establish the positivity of the quadratic form $u \mapsto \int \Delta u \Delta\left(u \frac{\varphi}{r_{c}^{N-\alpha}}\right)$ under suitable conditions. See Section 5.2.1 below.

Specializing Theorem 5.14 with the weight $v=v_{\epsilon}$ and $\delta^{2}=\frac{2 N-\alpha}{2(N-\alpha)}$, we can deduce (5.35) of Theorem 5.15 under a more restrictive hypothesis on the parameter $\alpha$. This is the reason why we do not deduce Theorem 5.15 as a consequence of Theorem 5.14 .

Proof of Theorem 5.15. We prove the claim for $u \in \mathscr{C}^{2}\left(\mathbb{R}^{N}\right)$. The general case follows by a regularization argument. By choosing $v=v_{\epsilon}$ in (5.26), and plugging in (5.11), we obtain

$$
\begin{aligned}
\int \Delta & u \Delta\left(u \frac{\varphi}{r_{\epsilon}^{N-\alpha}}\right) \geq \int\left(|\nabla u|^{2} p_{1}(t)+\left(\nabla u \cdot \frac{x}{|x|}\right)^{2} p_{2}(t)\right) \varphi \frac{r^{2}}{r_{\epsilon}^{N-\alpha+4}} \\
+ & \epsilon^{2}(t(N-2)+2(N-\alpha)) \int|\nabla u|^{2} \frac{\varphi}{r_{\epsilon}^{N-\alpha+4}}+\frac{1}{2} \int u^{2} \Delta^{2}\left(r_{\epsilon}^{\alpha-N}\right) \varphi \\
+ & 2(N-\alpha-t) E_{1}\left(\nabla u, \frac{r}{r_{\epsilon}^{N-\alpha+2}}, \varphi^{\prime}\right)+E_{2}\left(u, r_{\epsilon}^{\alpha-N}, \varphi\right),
\end{aligned}
$$

for any $t \in \mathbb{R}$, where

$$
p_{1}(t):=2(N-\alpha)+t(\alpha-4), \quad p_{2}(t):=t(2 N-2 \alpha+4-t)-2(N-\alpha)(N-\alpha+2) .
$$

Choosing $t=t_{\alpha}:=\frac{2 N-\alpha}{2}$, we get $p_{1}\left(t_{\alpha}\right) \geq 0$ (since the hypothesis on $\alpha$ ). By using (5.24) we complete the proof by taking into account that $p_{1}\left(t_{\alpha}\right)+p_{2}\left(t_{\alpha}\right)=C(N, \alpha) / 4$. 
The analog of Theorem 5.15 for singular weight is the following.

Theorem 5.17. Let $N>4$ and $\frac{2 N^{2}}{N+2} \geq \alpha \geq 4$ then for any $u \in \mathscr{C}^{2}\left(\mathbb{R}^{N}\right)$, and any nonnegative radial $\varphi \in \mathscr{C}_{0}^{4}\left(\mathbb{R}^{N}\right)$, we have

$$
\begin{aligned}
\int \Delta u \Delta\left(u \frac{\varphi}{|x|^{N-\alpha}}\right) \geq & \frac{C(N, \alpha)}{4} \int\left(\nabla u \cdot \frac{x}{|x|}\right)^{2} \frac{\varphi}{|x|^{N-\alpha+2}} \\
& +(N-\alpha)(N+2-\alpha) P\left(u^{2} \varphi, \alpha\right) \\
& -\alpha E_{1}\left(\nabla u, \frac{1}{|x|^{N-\alpha+1}}, \varphi^{\prime}\right)+E_{2}\left(u,|x|^{\alpha-N}, \varphi\right)
\end{aligned}
$$

where $C(N, \alpha), E_{1}, E_{2}$ and $P$ are defined in (5.36), (5.3), (5.27), and (5.32) respectively.

Proof. Since $\alpha \leq \frac{2 N^{2}}{N+2}$, we have $\epsilon^{2}((2 N-\alpha)(N-2)+4(N-\alpha)) \geq 0$, and from (5.35) we obtain

$$
\begin{aligned}
\int \Delta u \Delta\left(u \frac{\varphi}{r_{\epsilon}^{N-\alpha}}\right) \geq & \frac{1}{2} \int u^{2} \Delta^{2}\left(r_{\epsilon}^{\alpha-N}\right) \varphi \\
& +\frac{C(N, \alpha)}{4} \int\left(\nabla u \cdot \frac{x}{|x|}\right)^{2} \frac{r^{2}}{r_{\epsilon}^{N-\alpha+4}} \varphi \\
& -\alpha E_{1}\left(\nabla u, \frac{r}{r_{\epsilon}^{N-\alpha+2}}, \varphi^{\prime}\right)+E_{2}\left(u, r_{\epsilon}^{\alpha-N}, \varphi\right) .
\end{aligned}
$$

Letting $\epsilon \rightarrow 0$, we conclude the proof.

\subsubsection{Some results on functional positive quadratic forms}

The topic of this section is a brief detour from our main scope: namely the positivity of certain integral quadratic forms. From Theorem 5.17 we can deduce that

$$
\forall u \in \mathscr{C}_{0}^{2}\left(\mathbb{R}^{N}\right): \quad \int \Delta u \Delta\left(\frac{u}{|x|^{N-\alpha}}\right) \geq 0,
$$

for $\max \left\{4, \alpha_{1}\right\} \leq \alpha \leq N$ or $N+2 \leq \alpha \leq \alpha_{2}$. It remains to consider the case $N<\alpha<N+2$. In order to fill this gap, we need an extra argument based on the following asymptotic Hardy inequality.

In what follow, for brevity, we give only a sketch of the proofs of the results and we consider only smooth functions.

Theorem 5.18. Let $\gamma \in \mathbb{R}, u \in \mathscr{C}^{1}\left(\mathbb{R}^{N}\right)$. For any $\epsilon>0, t \in \mathbb{R}$ and a nonnegative radial function $\varphi \in \mathscr{C}_{0}^{1}\left(\mathbb{R}^{N}\right)$, we have

$$
\begin{aligned}
\int\left(\nabla u \cdot \frac{x}{|x|}\right)^{2} \frac{r^{2}}{r_{\epsilon}^{\gamma+2}} \varphi \geq & t(N-\gamma-2-t) \int u^{2} \frac{r^{2}}{r_{\epsilon}^{\gamma+4}} \varphi \\
& +\epsilon^{2} t(N-t) \int u^{2} \frac{1}{r_{\epsilon}^{\gamma+4}} \varphi+t E_{3}\left(u^{2}, \frac{r}{r_{\epsilon}^{\gamma+2}}, \varphi^{\prime}\right),
\end{aligned}
$$

and

$$
\begin{aligned}
\int\left(\nabla u \cdot \frac{x}{|x|}\right)^{2} \frac{1}{r_{\epsilon}^{\gamma}} \varphi \geq & t(N-\gamma-2-t) \int u^{2} \frac{r^{2}}{r_{\epsilon}^{\gamma+4}} \varphi \\
& +\epsilon^{2} t N \int u^{2} \frac{1}{r_{\epsilon}^{\gamma+4}} \varphi+t E_{3}\left(u^{2}, \frac{r}{r_{\epsilon}^{\gamma+2}}, \varphi^{\prime}\right),
\end{aligned}
$$

where

$$
E_{3}\left(u^{2}, \frac{r}{r_{\epsilon}^{\gamma+2}}, \varphi^{\prime}\right):=\int u^{2} \frac{r}{r_{\epsilon}^{\gamma+2}}\left(\nabla \varphi \cdot \frac{x}{|x|}\right)
$$


In particular, if $N-\gamma-2 \geq 0$, we have

$$
\int\left(\nabla u \cdot \frac{x}{|x|}\right)^{2} \frac{1}{r^{\gamma}} \varphi \geq \frac{(N-\gamma-2)^{2}}{4} \int u^{2} \frac{1}{r^{\gamma+2}} \varphi+\frac{N-\gamma-2}{2} E_{3}\left(u^{2}, \frac{1}{r^{\gamma+1}}, \varphi^{\prime}\right) .
$$

Finally, if $\varphi=\phi_{R}^{2}$, we have that

$$
\left|E_{3}\left(u^{2}, \frac{r}{r_{\epsilon}^{\gamma+2}}, \varphi^{\prime}\right)\right| \leq c_{1}\left(\phi_{1}\right) R^{N-\gamma-2} f_{A_{R}} u^{2} \phi_{R}
$$

The proof of the above theorem is based on an application of the vector field method (see [26]).

Proof. Let $t \neq 0$ and set $s:=\operatorname{sign}(t)$. Let $H$ be the vector field defined by $H(x):=s r_{\epsilon}^{-\gamma-2} \chi$. Since

$$
0=\int \operatorname{div}\left(H u^{2} \varphi\right)=\int \operatorname{div}(H) u^{2} \varphi+\int u^{2}(H \cdot \nabla \varphi)+2 \int u \varphi(H \cdot \nabla u),
$$

by computation we have

$$
\begin{gathered}
s(N-\gamma-2) \int u^{2} \frac{r^{2}}{r_{\epsilon}^{\gamma+4}} \varphi+\epsilon^{2} N s \int u^{2} \frac{1}{r_{\epsilon}^{\gamma+4}} \varphi+s \int u^{2} \frac{r}{r_{\epsilon}^{\gamma+2}}\left(\nabla \varphi \cdot \frac{x}{|x|}\right) \\
=-2 s \int u \varphi \frac{r}{r_{\epsilon}^{\gamma+2}}\left(\nabla u \cdot \frac{x}{|x|}\right) \leq \delta^{2} \int u^{2} \frac{1}{r_{\epsilon}^{\gamma+2}} \varphi+\delta^{-2} \int\left(\nabla u \cdot \frac{x}{|x|}\right)^{2} \frac{r^{2}}{r_{\epsilon}^{\gamma+2}} \varphi .
\end{gathered}
$$

Since

$$
\frac{1}{r_{\epsilon}^{\gamma+2}}=\frac{r^{2}}{r_{\epsilon}^{\gamma+4}}+\frac{\epsilon^{2}}{r_{\epsilon}^{\gamma+4}}
$$

by choosing $\delta^{2}=t s=|t|$, from (5.44), (5.45) and (5.42) we deduce (5.40).

The proof of (5.41) is similar to the proof of (5.40). Indeed, from the left hand side of (5.45) it follows that

$$
-2 s \int u \varphi \frac{r}{r_{\epsilon}^{\gamma+2}}\left(\nabla u \cdot \frac{x}{|x|}\right) \leq \delta^{2} \int u^{2} \frac{r^{2}}{r_{\epsilon}^{\gamma+4}} \varphi+\delta^{-2} \int\left(\nabla u \cdot \frac{x}{|x|}\right)^{2} \frac{1}{r_{\epsilon}^{\gamma}} \varphi .
$$

So we can proceed as above.

For $t \geq 0$, since the coefficient $\epsilon^{2} t N$ is nonnegative, in (5.41) we can ignore the term containing it, and hence by choosing $t=\frac{N-\gamma-2}{2}$, and letting $\epsilon \rightarrow 0$ in (5.41), we obtain (5.43).

Theorem 5.19. Let $u \in \mathscr{C}^{2}\left(\mathbb{R}^{N}\right), N>4$ and $\max \left\{4, \alpha_{1}\right\} \leq \alpha \leq \alpha_{2}$. For any nonnegative radial function $\varphi \in \mathscr{C}_{0}^{2}\left(\mathbb{R}^{N}\right)$, we have

$$
\begin{aligned}
\int \Delta u \Delta\left(u \frac{\varphi}{|x|^{N-\alpha}}\right) & \geq \frac{D_{1}}{16} \int u^{2} \frac{\varphi}{|x|^{N-\alpha+4}}-\alpha E_{1}\left(\nabla u, \frac{1}{|x|^{N-\alpha+1}}, \varphi^{\prime}\right) \\
+ & E_{2}\left(u,|x|^{\alpha-N}, \varphi\right)+\frac{C(N, \alpha)(\alpha-4)}{8} E_{3}\left(u^{2}, \frac{1}{|x|^{N-\alpha+3}}, \varphi^{\prime}\right),
\end{aligned}
$$

where

$$
D_{1}:=D_{1}(N, \alpha):=\alpha(\alpha-4)(2 N-\alpha)(2 N-\alpha-4),
$$

and for any $\epsilon>0$ we have

$$
\begin{aligned}
\int \Delta u \Delta\left(u \frac{\varphi}{r_{\epsilon}^{N-\alpha}}\right) \geq & \frac{1}{16} \int u^{2} \varphi \frac{D_{1} r^{4}+D_{2} \epsilon^{2} r^{2}+D_{3} \epsilon^{4}}{r_{\epsilon}^{N-\alpha+8}} \\
& -\alpha E_{1}\left(\nabla u, \frac{r}{r_{\epsilon}^{N-\alpha+2}}, \varphi^{\prime}\right)+E_{2}\left(u, r_{\epsilon}^{\alpha-N}, \varphi\right) \\
& +\frac{C(N, \alpha)(\alpha-4)}{8} E_{3}\left(u^{2}, \frac{r}{r_{\epsilon}^{N-\alpha+4}}, \varphi^{\prime}\right) \\
& +\frac{\epsilon^{2}}{2}((2 N-\alpha)(N-2)+4(N-\alpha)) t E_{3}\left(u^{2}, \frac{r}{r_{\epsilon}^{N-\alpha+6}}, \varphi^{\prime}\right),
\end{aligned}
$$


where $t \in \mathbb{R}$ and

$$
\begin{array}{r}
D_{2}:=2(\alpha-4)[N C(N, \alpha)+8(N-\alpha)(N+2-\alpha)(N+2)] \\
+8((2 N-\alpha)-2 \alpha)(\alpha-6-t) t, \\
D_{3}:=C(N, \alpha)(\alpha-4)(2 N-\alpha+4)+8(N-\alpha)(N+2-\alpha) N(N+2) \\
+8((2 N-\alpha)-2 \alpha) N t .
\end{array}
$$

Proof. Since $C(N, \alpha) \geq 0$, by choosing $\gamma=N-\alpha+2$ and plugging (5.43) in (5.38), we easily obtain (5.46).

Next we prove (5.47). Again, since $C(N, \alpha) \geq 0$, and $\epsilon^{2}((2 N-\alpha)(N-2)+4(N-\alpha)) \geq 0$, by inserting (5.40) in (5.35) with $\gamma=N-\alpha+2$ and $t=(\alpha-4) / 2$, and (5.41) in (5.35) with the choice $\gamma=N-\alpha+4$ and $t \in \mathbb{R}$, we obtain (5.47).

For compactly supported functions we have the following.

Corollary 5.20. Let $N=5$ and $4 \leq \alpha \leq 6$, or $N \geq 6$ and $\max \left\{4, \alpha_{1}\right\} \leq \alpha \leq \alpha_{2}$. Then we have

$$
\forall u \in \mathscr{C}_{0}^{2}\left(\mathbb{R}^{N}\right): \quad \int \Delta u \Delta\left(u \frac{1}{|x|^{N-\alpha}}\right) \geq 0 .
$$

Moreover if $N=5$ and $4 \leq \alpha<6, N=6$ and $4 \leq \alpha<8$, or $N \geq 7$ and $\max \left\{4, \alpha_{1}\right\} \leq \alpha \leq \alpha_{2}$,

$$
\forall u \in \mathscr{C}_{0}^{2}\left(\mathbb{R}^{N}\right) \quad u \neq 0: \quad \int \Delta u \Delta\left(u \frac{1}{|x|^{N-\alpha}}\right)>0 .
$$

Proof. Let $u \in \mathscr{C}_{0}^{2}\left(\mathbb{R}^{N}\right)$ and let $R>0$ be large enough such that the support of $u$ is contained in $B_{R}$. Let $\varphi=\phi_{R}$. With this choice, it follows that $E_{1}, E_{2}$ and $E_{3}$ in (5.46) vanish. This implies that

$$
\int \Delta u \Delta\left(u \frac{1}{|x|^{N-\alpha}}\right) \geq \frac{D_{1}}{16} \int \frac{u^{2}}{|x|^{N-\alpha+4}} .
$$

Now, if $N \geq 6$, we have $\max \left\{4, \alpha_{1}\right\} \leq \alpha \leq \alpha_{2} \leq 2 N-4<2 N$ and this implies that $D_{1}=$ $\alpha(\alpha-4)(2 N-\alpha)(2 N-\alpha-4)$ is nonnegative. Analogously, if $N=5$ and $4 \leq \alpha \leq 6<\alpha_{2}$, we conclude again that $D_{1} \geq 0$ and (5.48) holds.

In order to show the strict inequality in (5.49), we argue as follows. If $D_{1}>0$ the conclusion is obvious. It remains to analyze only the case when $\alpha=4$, and hence $N=5,6,7$. In this situation it follows that $C(N, 4)>0$ and then, the claim follows from Theorem 5.17.

For a non singular weight we have the following.

Corollary 5.21. Let $N=5$ and $4 \leq \alpha \leq 6$, or $N \geq 6$ and $\max \left\{4, \alpha_{1}\right\} \leq \alpha \leq \alpha_{2}$. Then we have

$$
\forall u \in \mathscr{C}_{0}^{2}\left(\mathbb{R}^{N}\right) \quad u \neq 0: \quad \int \Delta u \Delta\left(u \frac{1}{r_{\epsilon}^{N-\alpha}}\right)>0 .
$$

Proof. Let $u \in \mathscr{C}_{0}^{2}\left(\mathbb{R}^{N}\right)$, by using the same argument used in the proof of Corollary 5.20, from (5.47) we are reduced to study

$$
\int \Delta u \Delta\left(u \frac{\varphi}{r_{\epsilon}^{N-\alpha}}\right) \geq \frac{1}{16} \int u^{2} \varphi \frac{D_{1} r^{4}+2 D_{2} \epsilon^{2} r^{2}+D_{3} \epsilon^{4}}{r_{\epsilon}^{N-\alpha+8}},
$$

and hence to establish that $D_{1} r^{4}+2 D_{2} \epsilon^{2} r^{2}+D_{3} \epsilon^{4}$ is positive for some $t \in \mathbb{R}$. We note that the case $\alpha=N$ is trivial. Furthermore, from Remark 5.16 it follows that it is enough to perform the analysis only for $N<\alpha \leq N+2$ when $N \geq 6$ and for $5<\alpha \leq 6$ when $N=5$.

We begin analyzing the case $N \geq 7$. In this case the choice $t=0$ assures that $D_{1}, D_{2}, D_{3}>0$ and our claim is proved.

Let $N=6$ and $6<\alpha<8$. Even in this case the choice $t=0$ implies that $D_{1}, D_{2}, D_{3}>0$ and our claim holds. For the case $\alpha=8$, we have $D_{1}=0$ and $D_{2}, D_{3}>0$ for $t>0$ small enough $(0<t<\alpha-6)$ which implies (5.50). 
Let $N=5$. By the change of variable $\alpha=N+x$, we have to check the positivity of $D_{1}=$ $(x+5)(x+1)(1-x)(5-x), D_{2}=42 x^{3}-202 x^{2}+6 x+250+8 t^{2}(7 x-15)-8\left(7 x^{2}-22 x+15\right) t$, and $D_{3}=7 x^{4}-54 x^{3}+176 x^{2}-378 x+225-40 t(7 x-15)$, for $0<x \leq 1$ and for some $t \in \mathbb{R}$. The choice $t=1 / 8$ accomplishes the claim. We leave the detailed computations to the interested reader.

Remark 5.22. From the proof of (5.48)-(5.50), we can deduce the positivity of those quadratic forms, and that they can be controlled from below by quadratic integrals depending on $u$. For instance, if $u \in \mathscr{C}_{0}^{2}\left(\mathbb{R}^{N}\right)$ and $u \not \equiv 0$, if $D_{1}>0$ and $\max \left\{4, \alpha_{1}\right\}<\alpha \leq \alpha_{2}$. we have

$$
\int \Delta u \Delta\left(u \frac{1}{|x|^{N-\alpha}}\right) \geq \frac{D_{1}}{16} \int \frac{u^{2}}{|x|^{N-\alpha+4}}>0,
$$

while for $N>\alpha \geq 4$ and $\alpha>\alpha_{1}$, we have

$$
\int \Delta u \Delta\left(u \frac{1}{|x|^{N-\alpha}}\right) \geq \frac{C(N, \alpha)}{4} \int\left(\nabla u \cdot \frac{x}{|x|}\right)^{2} \frac{1}{|x|^{N-\alpha+2}}>0 .
$$

We leave the analysis of the remaining cases to the interested reader.

The results on the positivity of the quadratic form in Corollary 5.20 and 5.21 can be extended relaxing the request that the functions have compact support. The idea is to assume that for the functions $u$ the quantities $E_{1}\left(\nabla u,|x|^{\alpha-N-1}, \phi_{R}^{\prime}\right), E_{2}\left(u,|x|^{\alpha-N}, \phi_{R}\right)$ and $E_{3}\left(u^{2},|x|^{\alpha-N-3}, \phi_{R}^{\prime}\right)$ vanish as $R \rightarrow+\infty$. A sample of these kind of results is the following.

Theorem 5.23. Let $N=5$ and $4 \leq \alpha<6$, or $N=6$ and $4 \leq \alpha<8$, or $N \geq 7$ and $\max \left\{4, \alpha_{1}\right\} \leq \alpha \leq \alpha_{2}$. For any $u \in \mathscr{C}^{4}\left(\mathbb{R}^{N}\right), u \neq 0$ such that

$$
\begin{array}{ll}
\frac{\Delta^{2} u u}{|x|^{N-\alpha}} \in L^{1}\left(\mathbb{R}^{N}\right), & \limsup _{R \rightarrow+\infty} R^{\alpha} f_{A_{R}}|\Delta u|^{2}<\infty \\
\lim _{R \rightarrow+\infty} R^{\alpha-2} f_{A_{R}}|\nabla u|^{2}=0, & \lim _{R \rightarrow+\infty} R^{\alpha-4} f_{A_{R}} u^{2}=0,
\end{array}
$$

then

$$
\int \Delta u \Delta \frac{u}{|x|^{N-\alpha}} d x=\int \frac{\Delta^{2} u u}{|x|^{N-\alpha}} d x>0 .
$$

In particular, if (5.51) holds with $\alpha=4$, and $N=5,6,7$, then

$$
\int \Delta u \Delta \frac{u}{|x|^{N-4}} d x=\int \frac{\Delta^{2} u u}{|x|^{N-4}} d x>0 .
$$

Theorem 5.23 extends a result of [22] where the inequality (5.53) is proved for compactly supported functions. Indeed in [22] the author proves that (5.53) holds for $u \in \mathscr{C}_{0}^{\infty}\left(\mathbb{R}^{N}\right)$ and $N=5,6,7$, while in dimension $N \geq 8$, the inequality (5.53) is not satisfied. See also [23] for further extension to the higher order case.

\section{A priori estimates and Liouville theorems}

\subsection{A priori estimates}

In this section we deduce some a priori estimates on the solution of the inequality $\left(\mathbf{P}_{h}\right)$, that we remind for reader convenience

$$
-u \Delta^{2} u \geq h, \quad \text { on } \mathbb{R}^{N},
$$

whose definition of weak solution is given in (4.2). The following results refines some estimates obtaind earlier in [2]. 
Theorem 6.1. For any $d<1$ there exists $c_{d}=c(d)>0$ such that for any $h \in L_{\text {loc }}^{1}\left(\mathbb{R}^{N}\right)$ (without any assumption on its sign), for any weak solution $u \in H_{\text {loc }}^{2}\left(\mathbb{R}^{N}\right)$ of $\left(\mathbf{P}_{h}\right)$ and any nonnegative $\varphi \in \mathscr{C}_{0}^{2}\left(\mathbb{R}^{N}\right)$, we have

$$
\int_{\mathbb{R}^{N}} h \varphi+d \int_{\mathbb{R}^{N}}(\Delta u)^{2} \varphi \leq c_{d}\left[\int_{\mathbb{R}^{N}} u^{2} \frac{|\Delta \varphi|^{2}}{\varphi}+\int_{\mathbb{R}^{N}} u^{2}\left|\Delta \frac{|\nabla \varphi|^{2}}{\varphi}\right|+\int_{\mathbb{R}^{N}} u^{2} \frac{|\nabla \varphi|^{4}}{\varphi^{3}}\right] .
$$

In particular if $\varphi=\phi_{R}$, an admissible test function as in (1.8), there exists $c_{1}(d)=c_{1}\left(\phi_{1}, d\right)>0$ constant, and we have

$$
\begin{gathered}
\int_{\mathbb{R}^{N}} h \phi_{R}+d \int_{\mathbb{R}^{N}}(\Delta u)^{2} \phi_{R} \leq c_{1}(d) R^{-4} \int_{A_{R}} u^{2}, \\
\int_{\mathbb{R}^{N}}|\nabla u|^{2} \phi_{R} \leq c_{1} R^{-2} \int_{A_{R}} u^{2} \\
+\left(\int_{B_{2 R}} u^{2} \phi_{R}\right)^{1 / 2} \frac{1}{\sqrt{d}}\left(c_{1}(d) R^{-4} \int_{A_{R}} u^{2}-\int_{B_{2 R}} h \phi_{R}\right)^{1 / 2}, \\
\int_{A_{R}}|\nabla u|^{2} \leq c_{1} R^{-2} \int_{A_{R / 2} \cup A_{2 R}} u^{2} \\
+\left(\int_{A_{R / 2} \cup A_{R} \cup A_{2 R}} u^{2}\right)^{1 / 2} \frac{1}{\sqrt{d}}\left(c_{1}(d) R^{-4} \int_{A_{2 R}} u^{2}-\int_{B_{4 R}} h \phi_{2 R}\right)^{1 / 2} .
\end{gathered}
$$

If $h \geq 0$ on $B_{4 R}$ for some $R>0$, then

$$
\begin{aligned}
d \int_{B_{R}}(\Delta u)^{2} & \leq \int_{B_{R}} h \phi_{R}+d \int_{\mathbb{R}^{N}}(\Delta u)^{2} \phi_{R} \leq c_{1} R^{-4} \int_{A_{R}} u^{2}, \\
\int_{B_{R}}|\nabla u|^{2} & \leq c_{1} R^{-2} \int_{B_{2 R}} u^{2}, \\
\int_{A_{R}}|\nabla u|^{2} & \leq c_{1} R^{-2} \int_{A_{R / 2} \cup A_{R} \cup A_{2 R}} u^{2} .
\end{aligned}
$$

In particular if $h \geq c_{h}|u|^{q+1}$ for some $c_{h}>0$ and $q>1$, then there exists $c_{1}=c_{1}\left(c_{h}, q\right)>0$ such that for any $u \in H_{\text {loc }}^{2}\left(\mathbb{R}^{N}\right)$ weak solution of $\left(\mathbf{P}_{h}\right)$ and any $R>0$ there holds

$$
\begin{gathered}
c_{h}\left(f_{B_{R}}|u|^{2}\right)^{\frac{q+1}{2}} \leq c_{h} f_{B_{R}}|u|^{q+1} \leq f_{B_{R}} h+f_{B_{R}}(\Delta u)^{2} \leq c_{1} R^{-4 \frac{q+1}{q-1}}, \\
f_{B_{R}}|\nabla u|^{2} \leq c_{1} R^{-2 \frac{q+3}{q-1}} .
\end{gathered}
$$

Remark 6.2. Notice that the constant $c_{d}$ in the above theorem, does not depend on $u$ nor on $R$, and the quantity $c_{d} R^{-4} \int_{A_{R}} u^{2}-\int_{B_{2 R}} h \phi_{R}$ is nonnegative for any $R$ and any $u$.

The following Lemma contains an $H_{l o c}^{2}$ version of the identity

$$
\Delta \Phi^{2}=2 \Phi \Delta \Phi+2|\nabla \Phi|^{2} .
$$

Lemma 6.3. Let $\Phi \in H_{\text {loc }}^{2}(\Omega)$. For any $\varphi \in \mathscr{C}_{0}^{2}(\Omega)$, we have

$$
\int_{\Omega} \Phi^{2} \Delta \varphi=2 \int_{\Omega} \Phi \Delta \Phi \varphi+2 \int_{\Omega}|\nabla \Phi|^{2} \varphi,
$$


and

$$
\int_{\Omega}|\nabla \Phi|^{2} \varphi \leq \frac{1}{2} \int_{\Omega} \Phi^{2} \Delta \varphi+\left(\int_{\Omega} \Phi^{2}|\varphi|\right)^{1 / 2}\left(\int_{\Omega}|\Delta \Phi|^{2}|\varphi|\right)^{1 / 2} .
$$

Proof. Let $\left(m_{\eta}\right)_{\eta>0}$ be a family of standard mollifier cutoff functions. Setting

$$
u_{\eta}:=\Phi^{\star} m_{\eta},
$$

we have that $u_{\eta} \rightarrow \Phi, \nabla u_{\eta} \rightarrow \nabla \Phi$ and $\Delta u_{\eta} \rightarrow \Delta \Phi$ in $L_{l o c}^{2}\left(\mathbb{R}^{N}\right)$. Hence for any $\varphi \in \mathscr{C}_{0}^{2}\left(\mathbb{R}^{N}\right)$ we have

$$
2 \int_{\Omega} u_{\eta} \Delta u_{\eta} \varphi+2 \int_{\Omega}\left|\nabla u_{\eta}\right|^{2} \varphi=\int_{\Omega} \Delta u_{\eta}^{2} \varphi=\int_{\Omega} u_{\eta}^{2} \Delta \varphi .
$$

Letting $\eta \rightarrow 0$ identity (6.11) follows.

Inequality (6.12) can be deduced by applying Cauchy-Schwarz inequality to the identity (6.11).

\section{Proof of Theorem 6.1.}

Proof of estimate (6.1). By choosing $v \equiv 1$ in the identity (5.25), we obtain

$$
\begin{aligned}
\int h \varphi+\int(\Delta u)^{2} \varphi \leq-\int \Delta u \Delta & (u \varphi)+\int(\Delta u)^{2} \varphi \\
& =-\int \Delta u u \Delta \varphi-2 \int \Delta u(\nabla u, \nabla \varphi)=: I_{1}+I_{2} .
\end{aligned}
$$

Next, by Young inequality with $\epsilon>0$, we have

$$
\left|I_{1}\right| \leq \frac{\epsilon^{2}}{2} \int(\Delta u)^{2} \varphi+\frac{1}{2 \epsilon^{2}} \int u^{2} \frac{|\Delta \varphi|^{2}}{\varphi} .
$$

Analogously for any $\delta>0$ we have

$$
\left|I_{2}\right| \leq \delta^{2} \int(\Delta u)^{2} \varphi+\frac{1}{\delta^{2}} \int|\nabla u|^{2} \frac{|\nabla \varphi|^{2}}{\varphi} .
$$

For simplicity we denote by $\Theta$ the quantity $\Theta:=\frac{|\nabla \varphi|^{2}}{\varphi}$. Using the identity (6.11) with $\Phi=u$, integrating by parts and by Young's inequality, we obtain

$$
\begin{aligned}
\left|I_{2}\right| & \leq \delta^{2} \int(\Delta u)^{2} \varphi+\frac{1}{\delta^{2}} \int|\nabla u|^{2} \Theta \\
& =\delta^{2} \int(\Delta u)^{2} \varphi+\frac{1}{2 \delta^{2}} \int \Delta\left(u^{2}\right) \Theta-\frac{1}{\delta^{2}} \int u \Delta u \Theta \\
& =\delta^{2} \int(\Delta u)^{2} \varphi+\frac{1}{2 \delta^{2}} \int u^{2} \Delta \Theta-\frac{1}{\delta^{2}} \int u \Delta u \Theta \\
& \leq \delta^{2} \int(\Delta u)^{2} \varphi+\frac{1}{2 \delta^{2}} \int u^{2}|\Delta \Theta|+\frac{\gamma^{2}}{2 \delta^{2}} \int(\Delta u)^{2} \varphi+\frac{1}{2 \gamma^{2} \delta^{2}} \int u^{2} \frac{\Theta^{2}}{\varphi} .
\end{aligned}
$$

A suitable choice of the parameter $\epsilon, \delta$ and $\gamma$ gives the estimate (6.1).

Proof of (6.2). We begin noticing that each term in the right hand side of (6.1) has the form

$$
\int u^{2}|\psi|
$$

where $\psi$ stands for one of the functions $\frac{|\Delta \varphi|^{2}}{\varphi}, \Delta \frac{|\nabla \varphi|^{2}}{\varphi}$ or $\frac{|\nabla \varphi|^{4}}{\varphi^{3}}$.

Taking $\varphi:=\phi_{R}=\phi_{1}(|x| / R)$ an admissible test function the function, $|\psi|$ has support in $A_{R}$ and can be estimate as $|\psi| \leq c_{1} R^{-4}$. This concludes the proof of (6.2). 
Proof of (6.3). From (6.12) with $\Phi=u, \varphi=\phi_{R}$, and from the estimate of $|\Delta u|^{2}$, that can be deduced from (6.2), we have

$$
\begin{aligned}
& \int|\nabla u|^{2} \phi_{R} \leq \frac{1}{2} \int u^{2} \Delta \phi_{R}+\left(\int u^{2} \phi_{R}\right)^{1 / 2}\left(\int|\Delta u|^{2} \phi_{R}\right)^{1 / 2} \\
& \leq c_{1} R^{-2} \int_{A_{R}} u^{2}+\left(\int_{B_{2 R}} u^{2} \phi_{R}\right)^{1 / 2} \frac{1}{\sqrt{d}}\left(c_{1}(d) R^{-4} \int_{A_{R}} u^{2}-\int_{B_{2 R}} h \phi_{R}\right)^{1 / 2},
\end{aligned}
$$

that is the claim.

In order to prove estimate (6.4) we use (6.12) with $\Phi=u$ and as test function $\varphi(x)=\rho_{R}(|x|):=\phi_{2 R}(t)-\phi_{R / 2}(t)$. The support of $\rho_{R}$ is $G_{R}:=A_{R / 2} \cup A_{R} \cup A_{2 R}$. We have

$$
\begin{aligned}
\int_{A_{R}}|\nabla u|^{2} \leq & \int_{\mathbb{R}^{N}}|\nabla u|^{2} \rho_{R} \\
\leq & \int_{A_{R / 2} \cup A_{2 R}} u^{2}\left|\Delta \rho_{R}\right|+\left(\int_{G_{R}} u^{2} \rho_{R}\right)^{1 / 2}\left(\int_{G_{R}}|\Delta u|^{2} \rho_{R}\right)^{1 / 2} \\
\leq & c_{1} R^{-2} \int_{A_{R / 2} \cup A_{2 R}} u^{2}+\left(\int_{G_{R}} u^{2}\right)^{1 / 2}\left(\int_{B_{4 R}}|\Delta u|^{2} \phi_{2 R}\right)^{1 / 2} \\
\leq & c_{1} R^{-2} \int_{A_{R / 2} \cup A_{2 R}} u^{2} \\
& +\left(\int_{G_{R}} u^{2}\right)^{1 / 2} \frac{1}{\sqrt{d}}\left(c_{1}(d) R^{-4} \int_{A_{2 R}} u^{2}-\int_{B_{4 R}} h \phi_{2 R}\right)^{1 / 2},
\end{aligned}
$$

where in the last inequality we have used the estimate (6.2), obtaining the claim.

Estimates (6.5), (6.6) and (6.7) are an immediate consequence of (6.2), (6.3) (6.4) and the hypothesis $h \geq 0$.

Finally, to prove the missing inequalities (6.8) and (6.9) arguing again as in the proof of (6.2), and with the same notation, we notice that each term in the right hand side of (6.1) has the form (6.13). By using Hölder and Young inequalities, with exponent $x=\frac{q+1}{2}$, we obtain

$$
\int u^{2}|\psi| \leq\left(\int|u|^{q+1} \varphi\right)^{\frac{1}{x}}\left(\int \frac{|\psi|^{x^{\prime}}}{\varphi^{x^{\prime}-1}}\right)^{\frac{1}{x^{\prime}}} \leq \frac{\epsilon^{x}}{x c_{h}} \int h \varphi+\frac{1}{x^{\prime} \epsilon^{\chi^{\prime}}} \int \frac{|\psi|^{x^{\prime}}}{\varphi^{\chi^{\prime}-1}} .
$$

Taking $\varphi:=\phi_{R}=\phi_{1}(|x| / R)$ the term $\int \frac{|\psi|^{x^{\prime}}}{\varphi^{x^{\prime}-1}}$ behaves as $R^{N-4 x^{\prime}}=R^{N-4 \frac{q+1}{q-1}}$. Using (6.14) in (6.1), with a suitable choice of the parameter $d$ and $\epsilon$ we get

$$
\int_{B_{R}} h+\int_{B_{R}}(\Delta u)^{2} \leq c R^{N-4 \frac{q+1}{q-1}} \quad \text { and } f_{B_{R}}|\nabla u|^{2} \leq c R^{-2 \frac{q+3}{q-1}},
$$

which in turn, by our assumption $h \geq c_{h}|u|^{q+1}$ and Hölder inequality, yields (6.8). The inequality (6.9) is a consequence of the estimates (6.8) and (6.6).

\subsection{Some glimpses on Liouville theorems: weak solutions}

In this section we continue to study some Liouville theorems for weak solutions of the inequality,

$$
-u \Delta^{2} u \geq h, \quad \text { on } \mathbb{R}^{N},
$$


where $h \equiv 0$ or $h \geq|u|^{q+1}$.

In our first result we consider the homogeneous case.

Theorem 6.4. Let $u \in H_{l o c}^{2}\left(\mathbb{R}^{N}\right)$ be a solution of

$$
-u \Delta^{2} u \geq 0, \quad \text { on } \mathbb{R}^{N} .
$$

Assume that either,

$$
N=1, \ldots, 7, \quad \text { and } \quad \lim _{R \rightarrow \infty} f_{A_{R}}|u|^{2}=0
$$

or

$$
N \geq 8, \quad \text { and } \quad R^{\alpha_{1}-4} f_{A_{R}}|u|^{2} \leq C, \text { for any } R \text { large, }
$$

where $\alpha_{1}=\frac{2}{7}\left(3 N+2-\sqrt{2\left(N^{2}-N+2\right)}\right)$ is defined in (5.37), then $u \equiv 0$ a.e. in $\mathbb{R}^{N}$.

A first consequence of the above theorem is the following corollary which is reminiscent of a result proved by Ambrosio and Cabré (see [1] for details and applications).

Corollary 6.5. Let $u \in H_{\text {loc }}^{2}\left(\mathbb{R}^{N}\right)$ be a solution of (6.16) with $N \geq 5$. If for any $R$ large

$$
\int_{A_{R}}|u|^{2} \leq c R^{4},
$$

with c independent of $R$, then $u \equiv 0$ a.e. in $\mathbb{R}^{N}$.

Proof of Corollary 6.5. From the hypothesis (6.19), we have that

$$
f_{A_{R}}|u|^{2} \leq c R^{4-N} \rightarrow 0 \text { as } R \rightarrow \infty
$$

It is enough to check that the assumptions of Theorem 6.4 hold. Indeed, if $N=5,6,7$, then the hypothesis (6.17) is verified. While, for $N \geq 8$, since $\alpha_{1}<N$ it follows that

$$
R^{\alpha_{1}-4} f_{A_{R}}|u|^{2} \leq c R^{\alpha_{1}-4+4-N} \rightarrow 0 \text { as } R \rightarrow \infty,
$$

and (6.18) holds.

A further consequence of Theorem 6.4, under the stronger assumption of global integrability of the solutions of (6.16), is the following Liouville theorem that can be obtained directly by using the Hölder inequality.

Corollary 6.6. Let $u \in H_{\text {loc }}^{2}\left(\mathbb{R}^{N}\right) \cap L^{s}\left(\mathbb{R}^{N} \backslash B_{1}\right)$ be a solution of $(6.16)$. If $N=1, \ldots, 7$ and $2 \leq s<\infty$ or $N \geq 8$ and $2 \leq s \leq \frac{2 N}{\alpha_{1}-4}$, then $u \equiv 0$ a.e. in $\mathbb{R}^{N}$.

Notice that the expression

$$
\frac{2 N}{\alpha_{1}-4}=\frac{3 N^{2}-12 N+N \sqrt{2 N^{2}-2 N+4}}{N^{2}-10 N+20},
$$

as function of $N$ is decreasing and converges to $3+\sqrt{2}$ as $N \rightarrow+\infty$.

An essential ingredient of the proof of Theorem 6.4 is the following.

Lemma 6.7. Let $u$ be an harmonic function in $\mathbb{R}^{N}$. Then the following inequality holds

$$
f_{B_{R}}|u-u(0)|^{2} \leq c f_{A_{R}} u^{2},
$$

where $c>0$ does not depend on $u$ nor $R$. 
In particular if

$$
\liminf _{R \rightarrow \infty} \int_{A_{R}} u^{2}=0
$$

then $u \equiv 0$ in $\mathbb{R}^{N}$.

Proof. Let $\phi_{R}$ be defined in (1.8). Since $u$ is harmonic, from (6.10), we have

$$
\int_{B_{R}}|\nabla u|^{2} \leq \int|\nabla u|^{2} \phi_{R}=\frac{1}{2} \int u^{2} \Delta \phi_{R} \leq c_{1} R^{-2} \int_{A_{R}} u^{2} .
$$

Next by Poincaré inequality, setting $u_{B_{R}}=f_{B_{R}} u$. we have

$$
\int_{B_{R}}\left|u-u_{B_{R}}\right|^{2} \leq c R^{2} \int_{B_{R}}|\nabla u|^{2} \leq c \int_{A_{R}} u^{2} .
$$

Now by using the fact that $u$ has the mean value property, that is $u(0)=f_{B_{R}} u$, we obtain (6.20).

Next, we observe that for an harmonic function $u$, for any $x \in \mathbb{R}^{N}$, we have

$$
u(x)=f_{B_{2 R}(x) \backslash B_{R}(x)} u .
$$

Indeed, by the mean value property, we have

$$
\begin{aligned}
f_{B_{2 R}(x) \backslash B_{R}(x)} u & =\frac{1}{\omega_{N} R^{N}\left(2^{N}-1\right)}\left(\int_{B_{2 R}(x)} u-\int_{B_{R}(x)} u\right) \\
& =\frac{2^{N}}{2^{N}-1} f_{B_{2 R}(x)} u-\frac{1}{2^{N}-1} f_{B_{R}(x)} u \\
& =\frac{2^{N}}{2^{N}-1} u(x)-\frac{1}{2^{N}-1} u(x)=u(x) .
\end{aligned}
$$

Next, from the inequalities

$$
|u(0)|=\left|f_{A_{R}} u\right| \leq f_{A_{R}}|u| \leq\left(f_{A_{R}}|u|^{2}\right)^{1 / 2},
$$

and (6.21), we deduce that $u(0)=0$. Finally, from (6.20) and (6.21), we have

$$
f_{B_{R}} u^{2} \leq c f_{A_{R}} u^{2} \rightarrow 0 \text { as } R \rightarrow \infty .
$$

Now since $u^{2}$ is subharmonic, we know that

$$
f_{B_{R}} u^{2} \pi \sup _{\mathbb{R}^{N}} u^{2} \text { as } R \rightarrow \infty
$$

and we deduce $u^{2} \equiv 0$.

Proof of of Theorem 6.4. Let $u$ be a solution of (6.16). Set $\alpha:=4$ if $N=1, \ldots, 7$ and $\alpha:=\alpha_{1}$ in the remaining cases $N \geq 8$.

We begin proving the claim under the hypothesis

$$
\lim _{R \rightarrow \infty} R^{\alpha-4} f_{A_{R}}|u|^{2}=0
$$

First we examine the cases $N=1,2,3$, 4. From (6.2) it follows that

$$
\int(\Delta u)^{2} \phi_{R} \leq c R^{-4} \int_{A_{R}} u^{2}=c R^{N-4} f_{A_{R}} u^{2} \text {. }
$$


Since $\alpha=4$, by our assumption (6.23) it follows that by letting $R \rightarrow \infty$, we deduce that $u$ is an harmonic function and Lemma 6.7 applies.

Next we consider the cases $N \geq 5$. An application of (5.38), taking into account that $C(N, \alpha) \geq 0$, yields

$$
\begin{aligned}
0 \geq \int \Delta u \Delta\left(u \frac{\varphi}{|x|^{N-\alpha}}\right) \geq & \frac{C(N, \alpha)}{4} \int\left(\nabla u \cdot \frac{x}{|x|}\right)^{2} \frac{\varphi}{|x|^{N-\alpha+2}} \\
& +(N-\alpha)(N+2-\alpha) P\left(u^{2} \varphi, \alpha\right) \\
& -\alpha E_{1}\left(\nabla u, \frac{1}{|x|^{N-\alpha+1}}, \varphi^{\prime}\right)+E_{2}\left(u,|x|^{\alpha-N}, \varphi\right) .
\end{aligned}
$$

By using (6.23) in (6.7) and (6.5), we deduce

$$
\lim _{R \rightarrow \infty} R^{\alpha-2} f_{A_{R}}|\nabla u|^{2}=0 \text { and } \lim _{R \rightarrow \infty} R^{\alpha} f_{A_{R}}|\Delta u|^{2}=0 .
$$

Next by choosing $\varphi=\phi_{R}^{4}$ as in (1.8), from estimate (5.4) and (5.29) we deduce that $E_{1} \rightarrow 0$ and $E_{2} \rightarrow 0$ as $R \rightarrow \infty$.

Since $P\left(u^{2} \phi_{R}^{4}, \alpha\right)$ is nonnegative and non decreasing with respect to $R$, by the monotone convergence theorem we obtain $P\left(u^{2} \phi_{R}^{4}, \alpha\right) \rightarrow P\left(u^{2}, \alpha\right) \geq 0$ as $R \rightarrow \infty$. Finally, from (6.24) we have

$$
\begin{aligned}
0 & \geq \int \Delta u \Delta\left(u \frac{1}{|x|^{N-\alpha}}\right) \\
& \geq \frac{C(N, \alpha)}{4} \int\left(\nabla u \cdot \frac{x}{|x|}\right)^{2} \frac{1}{|x|^{N-\alpha+2}}+(N-\alpha)(N+2-\alpha) P\left(u^{2}, \alpha\right) \geq 0,
\end{aligned}
$$

hence

$$
\int \Delta u \Delta\left(u \frac{1}{|x|^{N-\alpha}}\right)=\frac{C(N, \alpha)}{4} \int\left(\nabla u \cdot \frac{x}{|x|}\right)^{2} \frac{1}{|x|^{N-\alpha+2}}=P\left(u^{2}, \alpha\right)=0 .
$$

In the case $\alpha=\alpha_{1}>4$, from the definition of $P$ in (5.32), it follows that $u^{2} \equiv 0$. This complete the proof in the case $\alpha_{1}>4$.

Next we consider the case $\alpha=4$. Since $C(N, 4)>0$ we get

$$
\int\left(\nabla u \cdot \frac{x}{|x|}\right)^{2} \frac{1}{|x|^{N-\alpha+2}}=0,
$$

that is $\left(\nabla u \cdot \frac{x}{|x|}\right)=0$ on $\mathbb{R}^{N}$. Therefore $u$ is a constant function whose mean vanishes at infinity, that is $u \equiv 0$.

Finally we consider the cases when $N \geq 8$ and (6.18) holds. Arguing as above, using (6.18) in (6.7) and (6.5), we deduce that

$$
R^{\alpha_{1}-2} f_{A_{R}}|\nabla u|^{2} \leq C \text { and } R^{\alpha_{1}} f_{A_{R}}|\Delta u|^{2} \leq C .
$$

By choosing $\varphi=\phi_{R}^{4}$ as in (1.8), from estimate (5.4) and (5.29) it follows that $\left|E_{1}\right| \leq c$ and $\left|E_{2}\right| \leq c$ for some constant $c>0$. Since $C\left(N, \alpha_{1}\right)=0$, from (5.46) we have

$$
c \geq \int \Delta u \Delta\left(u \frac{\phi_{R}^{4}}{|x|^{N-\alpha}}\right) \geq(N-\alpha)(N+2-\alpha) P\left(u^{2} \phi_{R}^{2}, \alpha_{1}\right),
$$

and by letting $R \rightarrow \infty$, we obtain

$$
c \geq P\left(u^{2}, \alpha_{1}\right)=\frac{\left(\alpha_{1}-2\right)\left(\alpha_{1}-4\right)}{2} \int_{\mathbb{R}^{N}} u^{2} \frac{1}{|x|^{N-\alpha_{1}+4}}
$$

that is $\frac{u^{2}}{|x|^{N-\alpha_{1}+4}} \in L^{1}\left(\mathbb{R}^{N}\right)$.

Therefore,

$$
R^{\alpha_{1}-4} f_{A_{R}}|u|^{2} \leq C \int_{A_{R}} u^{2} \frac{1}{|\chi|^{N-\alpha_{1}+4}} \rightarrow 0, \quad \text { as } R \rightarrow \infty .
$$


That is $u$ satisfies the stronger assumption (6.23), and the claim follows.

One of the main result of this paper within the class of weak solutions is the following.

Theorem 6.8. Let $u \in H_{l o c}^{2}\left(\mathbb{R}^{N}\right) \cap L_{\text {loc }}^{q+1}\left(\mathbb{R}^{N}\right)$ be a solution of

$$
-u \Delta^{2} u \geq c|u|^{q+1} \quad \text { on } \mathbb{R}^{N}
$$

If

$$
\begin{gathered}
N=1, \ldots, 7, \quad \text { and } q>1 \\
\text { or } \\
N \geq 8 \text { and } 1<q \leq q_{N}
\end{gathered}
$$

where

$$
\begin{aligned}
q_{N} & :=\frac{\alpha_{1}+4}{\alpha_{1}-4}=\frac{3 N+16-\sqrt{4-2 N+2 N^{2}}}{3 N-12-\sqrt{4-2 N+2 N^{2}}} \\
& =\frac{N^{2}+2 N-28+4 \sqrt{4-2 N+2 N^{2}}}{N^{2}-10 N+20},
\end{aligned}
$$

then $u \equiv 0$ a.e. in $\mathbb{R}^{N}$.

Proof. We distinguish various cases. Let $N=1, \ldots, 4$. Under this assumption the claim follows directly form (6.8). Indeed, we have

$$
\int_{B_{R}}|u|^{q+1}+\int_{B_{R}}(\Delta u)^{2} \leq C R^{N-4 \frac{q+1}{q-1}} \rightarrow 0 \quad \text { as } R \rightarrow+\infty .
$$

Next we consider the case $N \geq 5$. From (6.8), we deduce that

$$
R^{\alpha-4} f_{A_{R}}|u|^{2} \leq R^{\alpha-4 \frac{q+1}{q-1}}
$$

Now if $N=5,6,7$, the choice $\alpha=4$ is admissible in Theorem 6.4 and since $4-4 \frac{q+1}{q-1}<0$, the claim follows.

Let $N \geq 8$. In this case, $\alpha=\alpha_{1}>4$ is an admissible choice in Theorem 6.4 and since $\alpha_{1}-4 \frac{q+1}{q-1} \leq 0$, we conclude the proof.

\subsection{Weighted a priori estimates}

In this section we shall prove some a priori estimates for solutions of

$$
-u \Delta^{2} u \geq h, \quad \text { on } \mathbb{R}^{N} .
$$

These estimates will be useful in the study of distributional solutions of the fourth order problem $\left(g \mathbf{P}_{f}\right)$. Notice that there is no hypothesis on the sign of the function $h$.

Theorem 6.9. Let $u \in \mathscr{C}^{4}\left(\mathbb{R}^{N}\right)$ and $h \in L_{\text {loc }}^{\infty}\left(\mathbb{R}^{N}\right)$ satisfy $\left(\mathbf{P}_{h}\right)$. Let $\alpha \geq 4, N>4$.

For any nonnegative and radial function $\varphi \in \mathscr{C}_{0}^{4}\left(\mathbb{R}^{N}\right)$, we have

$$
\begin{aligned}
2(N-\alpha)(N+2 & -\alpha) \int\left(\nabla u \cdot \frac{x}{|x|}\right)^{2} \frac{\varphi}{|x|^{N-\alpha+2}} \geq \int h \frac{\varphi}{|x|^{N-\alpha}} \\
& +\int(\Delta u)^{2} \frac{\varphi}{|x|^{N-\alpha}}+2(N-\alpha) \int|\nabla u|^{2} \frac{\varphi}{|x|^{N-\alpha+2}} \\
& +(N-\alpha)(N+2-\alpha) P\left(u^{2} \varphi, \alpha\right) \\
+ & 2(N-\alpha) E_{1}\left(\nabla u, \frac{1}{|x|^{N-\alpha+1}}, \varphi^{\prime}\right)+E_{2}\left(u,|x|^{\alpha-N}, \varphi\right),
\end{aligned}
$$


and for $N+\sqrt{N^{2}-4 N} \geq \alpha \geq 4$, for any $\epsilon>0$ by setting $r:=|x|, r_{\epsilon}:=\left(\epsilon^{2}+|x|^{2}\right)^{1 / 2}$, we have,

$$
\begin{aligned}
0 \geq & \int h \frac{\varphi}{r_{\epsilon}^{N-\alpha}}+\frac{C(N, \alpha)}{4} \int\left(\nabla u \cdot \frac{x}{|x|}\right)^{2} \frac{r^{2}}{r_{\epsilon}^{N-\alpha+4}} \varphi+\frac{1}{2} \int u^{2} \Delta^{2}\left(r_{\epsilon}^{\alpha-N}\right) \varphi \\
& -\alpha E_{1}\left(\nabla u, \frac{r}{r_{\epsilon}^{N-\alpha+2}}, \varphi^{\prime}\right)+E_{2}\left(u, r_{\epsilon}^{\alpha-N}, \varphi\right) .
\end{aligned}
$$

Furthermore, for any $\delta>0$, let $\phi_{1}$ be an admissible test function, then there exists $c_{1}=c\left(\phi_{1}, \delta\right)$ such that for any $R$ large, by setting $\varphi=\phi_{R}^{4}$ we have

$$
\begin{aligned}
&\left|E_{1}\left(\nabla u, \frac{r}{r_{\epsilon}^{N-\alpha+2}}, \varphi^{\prime}\right)\right| \leq c_{1}(\delta) R^{\alpha-4} f_{B_{2 R}} u^{2} \phi_{R}+c_{1}(\delta) R^{\alpha-4} f_{A_{R}} u^{2}-\delta^{2} R^{\alpha} f_{B_{2 R}} h \phi_{R}, \\
& \mid E_{1}\left(\nabla u, \frac{r}{\left.r_{\epsilon}^{N-\alpha+2}, \varphi^{\prime}\right) \mid \leq c_{1}(\delta) R^{\alpha-4} f_{A_{R / 2} \cup A_{R} \cup A_{2 R}} u^{2}-\delta^{2} R^{\alpha} f_{B_{4 R}} h \phi_{2 R},}\right. \\
&\left|E_{2}\left(u, r_{\epsilon}^{\alpha-N}, \varphi\right)\right| \leq c_{1}(\delta) R^{\alpha-4} f_{B_{2 R}} u^{2} \phi_{R}+c_{1}(\delta) R^{\alpha-4} f_{A_{R}} u^{2} \\
&-\delta^{2} R^{\alpha} f_{B_{2 R}} h \phi_{R}, \\
&\left|E_{2}\left(u, r_{\epsilon}^{\alpha-N}, \varphi\right)\right| \leq c_{1}(\delta) R^{\alpha-4} f_{A_{R / 2} \cup A_{R} \cup A_{2 R}} u^{2}-\delta^{2} R^{\alpha} f_{B_{4 R}} h \phi_{2 R},
\end{aligned}
$$

and the involved constants do not depend on $\epsilon$ nor on $u$.

Proof. We begin by observing that if $u$ solves $\left(\mathbf{P}_{h}\right)$, then for any nonnegative test function $\varphi$, we have

$$
\int-\Delta u \Delta\left(u v_{\epsilon} \varphi\right) \geq \int h v_{\epsilon} \varphi .
$$

where $v_{\epsilon}$ is defined in (5.9). Now letting $\epsilon \rightarrow 0$, an application of the identity (5.31) yields (6.27), while from the inequality (5.35) we deduce (6.28).

In order to estimate $E_{1}$, from (5.4), it suffices to estimate $R^{\alpha-2} f_{A_{R}}|\nabla u|^{2} \phi_{R}$. To this end from the estimate (6.3) with $d=1 / 2$, and by Young's inequality, we deduce

$$
\begin{aligned}
& R^{-2} f_{\mathbb{R}^{N}}|\nabla u|^{2} \phi_{R} \leq c_{1} R^{-4} f_{A_{R}} u^{2} \\
& \quad+\left(R^{-4} f_{B_{2 R}} u^{2} \phi_{R}\right)^{1 / 2} \sqrt{2}\left(c_{1} R^{-4} f_{A_{R}} u^{2}-f_{B_{2 R}} h \phi_{R}\right)^{1 / 2} \\
& \leq c_{1} R^{-4} f_{A_{R}} u^{2} \\
& \quad+\frac{1}{2 \delta^{2}} R^{-4} f_{B_{2 R}} u^{2} \phi_{R}+c_{1} \delta^{2} R^{-4} f_{A_{R}} u^{2}-\delta^{2} f_{B_{2 R}} h \phi_{R},
\end{aligned}
$$

that is the estimate (6.29). The estimate (6.30) follows similarly from (6.4).

To estimate $E_{2}$, it suffices to estimate the terms in (5.29) containing $\Delta u$ and $\nabla u$. Arguing as before, by Young's inequality and the estimate (6.2) with $d=1 / 2$, we have

$$
\begin{array}{r}
\left(f_{A_{R}}|\Delta u|^{2} \phi_{R}\right)^{\frac{1}{2}}\left(R^{-4} f_{A_{R}}|u|^{2}\right)^{\frac{1}{2}} \leq \frac{\delta^{2}}{2} f_{A_{R}}|\Delta u|^{2} \phi_{R}+\frac{1}{2 \delta^{2}} R^{-4} f_{A_{R}}|u|^{2} \\
\leq \delta^{2} c_{1} R^{-4} f_{A_{R}}|u|^{2}-\delta^{2} f h \phi_{R}+\frac{1}{2 \delta^{2}} R^{-4} f_{A_{R}}|u|^{2} .
\end{array}
$$

The last term in the estimate (5.29) of $E_{2}$, can be controlled similarly by using (6.33) where $\delta$ is replaced by $\eta$, obtaining

$$
\left(f_{A_{R}}|\Delta u|^{2} \phi_{R}\right)^{\frac{1}{2}}\left(R^{-2} f_{A_{R}}|\nabla u|^{2} \phi_{R}\right)^{\frac{1}{2}} \leq
$$




$$
\begin{aligned}
& \leq \frac{\delta^{2}}{2} f_{A_{R}}|\Delta u|^{2} \phi_{R}+\frac{1}{2 \delta^{2}} R^{-2} f_{A_{R}}|\nabla u|^{2} \phi_{R} \\
& \leq \delta^{2} c_{1} R^{-4} f_{A_{R}}|u|^{2}-\delta^{2} f h \phi_{R}+\frac{1}{4 \delta^{2} \eta^{2}} R^{-4} f_{B_{2 R}} u^{2} \phi_{R} \\
& \quad+c_{1} \frac{1+\eta^{2}}{2 \delta^{2}} R^{-4} f_{A_{R}} u^{2}-\frac{\eta^{2}}{2 \delta^{2}} f_{B_{2 R}} h \phi_{R} .
\end{aligned}
$$

Now, choosing $\eta^{2}=2 \delta^{4}$, gluing together the estimates (5.29), (6.34) and (6.35) and rescaling $\delta$ we get the estimate (6.31). Similarly we deduce (6.32), concluding the proof.

Corollary 6.10. Let $N>4$ and $\alpha \geq 4$ such that $C(N, \alpha) \geq 0$. Then for any $\delta>0$ and any admissible function $\phi_{1}$, there exists $c_{1}(\delta)>0$ such that for any $u \in \mathscr{C}^{4}\left(\mathbb{R}^{N}\right)$ and $h \in L_{\text {loc }}^{\infty}\left(\mathbb{R}^{N}\right)$ satisfying $\left(\mathbf{P}_{h}\right)$, and any $R$ large, we have

$$
\begin{aligned}
& c_{1}(\delta) R^{\alpha-4} f_{B_{2 R}} u^{2} \phi_{R}+c_{1}(\delta) R^{\alpha-4} f_{A_{R}} u^{2} \\
& \geq \delta^{2} R^{\alpha} f_{B_{2 R}} h \phi_{R}+\int h \frac{\phi_{R}}{|x|^{N-\alpha}}+(N-\alpha)(N+2-\alpha) P\left(u^{2} \phi_{R}^{4}, \alpha\right),
\end{aligned}
$$

where $\phi_{R}$ is defined as in (1.8).

Notice that the last inequality is an estimate on the possible solutions of $\left(\mathbf{P}_{h}\right)$ which does not involve the derivatives of $u$. This will be useful when dealing with distributional solutions.

\subsection{Distributional solutions: a priori estimates}

The a priori estimates contained in the following result will play a crucial role in what follows.

Theorem 6.11. Assume that $f, g: \mathbb{R} \rightarrow \mathbb{R}$ are continuous functions satisfying

$$
f(t) t \geq 0, g(t) t \geq 0, \quad \text { for all } t \in \mathbb{R},
$$

and set $H$ as in (1.6), that is

$$
H(t):= \begin{cases}f(t) t, & \text { for } t \geq 0, \\ g(t) t, & \text { for } t<0 .\end{cases}
$$

Let $u \in L_{\text {loc }}^{s}\left(\mathbb{R}^{N}\right)$ be such that $f(u), g(u) \in L_{\text {loc }}^{s^{\prime}}\left(\mathbb{R}^{N}\right)$ with $2 \leq s \leq+\infty$, and let $u$ be a distributional solution of

$$
g(u) \geq-\Delta^{2} u \geq f(u) \quad \text { on } \mathbb{R}^{N} .
$$

Then,

1. For any $R$ we have

$$
\int_{B_{R}} H(u) \leq c R^{-4} \int_{A_{R}} u^{2} .
$$

2. Let $N>\alpha \geq 4$ be such that $C(N, \alpha) \geq 0$, we have that $|x|^{\alpha-N} H(u) \in L_{\text {loc }}^{1}\left(\mathbb{R}^{N}\right)$, and for any $R$, we have

$$
\begin{gathered}
\int_{B_{R}} H(u) \frac{1}{r_{\epsilon}^{N-\alpha}}+\frac{1}{2} \int_{B_{R}} u^{2} \Delta^{2}\left(r_{\epsilon}^{\alpha-N}\right) \leq c R^{\alpha-4} f_{A_{R / 2} \cup A_{R} \cup A_{2 R}} u^{2}, \\
\int_{B_{R}} H(u) \frac{1}{|x|^{N-\alpha}} \leq c R^{\alpha-4} f_{A_{R / 2} \cup A_{R} \cup A_{2 R}} u^{2}, \\
\int_{B_{R}} H(u) \frac{1}{|x|^{N-\alpha}} \leq c R^{\alpha-4} f_{B_{2 R}} u^{2} .
\end{gathered}
$$


3. If $H(t) \geq c_{q}|t|^{q+1}$ for some $q>1$ and $c_{q}>0$, we have

$$
f_{B_{R}} H(u) \leq c R^{-4 \frac{q+1}{q-1}}, \quad f_{B_{R}} u^{2} \leq c R^{-\frac{8}{q-1}} .
$$

Hence, if $N>\alpha \geq 4$ and $C(N, \alpha) \geq 0$, it follows that

$$
\int_{B_{R}} H(u) \frac{1}{r_{\epsilon}^{N-\alpha}}+\frac{1}{2} \int_{B_{R}} u^{2} \Delta^{2}\left(r_{\epsilon}^{\alpha-N}\right) \leq c R^{\alpha-4 \frac{q+1}{q-1}}
$$

(where $c>0$ is independent of $u$ and $R$ ).

Proof. Let $\left(m_{n}\right)_{n>0}$ be a family of standard radial mollifier. By using $m_{n}(x-\cdot)$ as test function for $\left({ }_{g} \mathbf{P}_{f}\right)$, and setting

$$
u_{n}:=u \star m_{n}, \quad f_{n}:=f(u) \star m_{n}, \quad g_{n}:=g(u) \star m_{n},
$$

we shall deal with a sequence of functions $u_{n}, f_{n}$ and $g_{n}$ satisfying the following properties

$$
\begin{gathered}
u_{n} \rightarrow u \quad \text { in } L_{\text {loc }}^{s}\left(\mathbb{R}^{N}\right) \cap L_{\text {loc }}^{2}\left(\mathbb{R}^{N}\right) \text { and a.e. as } n \rightarrow+\infty \\
f_{n} \rightarrow f(u), \quad g_{n} \rightarrow g(u) \quad \text { in } L_{\text {loc }}^{s^{\prime}}\left(\mathbb{R}^{N}\right) \text { and a.e. as } n \rightarrow+\infty .
\end{gathered}
$$

Since the functions $t \mapsto t^{+}$and $t \mapsto t^{-}$are Lipschitz functions we have (up to a subsequence) that

$$
u_{n}^{ \pm} \rightarrow u^{ \pm} \text {in } L_{\text {loc }}^{s}\left(\mathbb{R}^{N}\right) \cap L_{\text {loc }}^{2}\left(\mathbb{R}^{N}\right) \text { and a.e. as } n \rightarrow+\infty \text {. }
$$

Therefore $u_{n}$ is a smooth functions satisfying

$$
g_{n} \geq-\Delta^{2} u_{n} \geq f_{n}, \quad \text { on } \mathbb{R}^{N} .
$$

Multiplying by $u_{n}^{+}$, then by $-u_{n}^{-}$and then adding the inequalities we have

$$
g_{n} u_{n}^{+}-f_{n} u_{n}^{-} \geq-u_{n} \Delta^{2} u_{n} \geq f_{n} u_{n}^{+}-g_{n} u_{n}^{-} .
$$

That is, setting $h_{n}:=f_{n} u_{n}^{+}-g_{n} u_{n}^{-}$, it follows that $u_{n}$ is a smooth solution of

$$
-u_{n} \Delta^{2} u_{n} \geq h_{n},
$$

and hence all the a priori estimates of the previous sections apply. Before to going on, let us to notice that

$$
h_{n} \rightarrow f(u) u^{+}-g(u) u^{-}=H(u) \text { in } L_{l o c}^{1}\left(\mathbb{R}^{N}\right) \text { and a.e. }
$$

and furthermore, since $g(u)\left(-u^{-}\right) \geq 0$ we get that

$$
h_{n} \rightarrow H(u)=f(u) u^{+}-g(u) u^{-} \geq 0 .
$$

Applying Theorem (6.1) to inequality (6.42), from (6.2) with $d=1 / 2$, we deduce that,

$$
\int_{\mathbb{R}^{N}} h_{n} \phi_{R} \leq c_{1} R^{-4} \int_{A_{R}} u_{n}^{2} .
$$

Letting $n \rightarrow+\infty$, and taking into account (6.43) we have (6.36).

Next, since $C(N, \alpha) \geq 0$, from (6.28) we have

$$
\begin{aligned}
& \int h_{n} \frac{\phi_{R}}{r_{\epsilon}^{N-\alpha}}+\frac{1}{2} \int u_{n}^{2} \Delta^{2}\left(r_{\epsilon}^{\alpha-N}\right) \phi_{R} \\
& \quad \leq \alpha\left|E_{1}\left(\nabla u_{n}, \frac{r}{r_{\epsilon}^{N-\alpha+2}}, \phi_{R}^{\prime}\right)\right|+\left|E_{2}\left(u_{n}, r_{\epsilon}^{\alpha-N}, \phi_{R}\right)\right| .
\end{aligned}
$$


By using the estimates (6.30) and (6.32) with $\delta=1$ we have

$$
\begin{aligned}
& \int h_{n} \frac{\phi_{R}}{r_{\epsilon}^{N-\alpha}}+\frac{1}{2} \int u_{n}^{2} \Delta^{2}\left(r_{\epsilon}^{\alpha-N}\right) \phi_{R} \leq \\
& \quad \leq(N+1)\left(c_{1} R^{\alpha-4} f_{A_{R / 2} \cup A_{R} \cup A_{2 R}} u_{n}^{2}-2 R^{\alpha} f_{B_{4 R}} h_{n} \phi_{2 R}\right) .
\end{aligned}
$$

Letting $n \rightarrow \infty$, and taking into account (6.43), we deduce

$$
\begin{aligned}
\int_{B_{R}} H & (u) \frac{1}{r_{\epsilon}^{N-\alpha}}+\frac{1}{2} \int u^{2} \Delta^{2}\left(r_{\epsilon}^{\alpha-N}\right) \phi_{R} \\
& \leq(N+1)\left(c_{1} R^{\alpha-4} f_{A_{R / 2} \cup A_{R} \cup A_{2 R}} u^{2}-R^{\alpha} f_{B_{4 R}} H(u) \phi_{2 R}\right) \\
& \leq c R^{\alpha-4} f_{A_{R / 2} \cup A_{R} \cup A_{2 R}} u^{2},
\end{aligned}
$$

that is (6.37). Estimate (6.38) follows taking into account that $\Delta^{2}\left(r_{\epsilon}^{\alpha-N}\right) \geq 0$, and by the monotone convergence theorem by letting $\epsilon \rightarrow 0$.

The proof of (6.39) follows similarly to (6.37), from (6.45), by using estimates (6.29) and (6.31) with $\delta=1$, obtaining

$$
\begin{aligned}
\int h_{n} \frac{\phi_{R}}{r_{\epsilon}^{N-\alpha}} & +\frac{1}{2} \int u_{n}^{2} \Delta^{2}\left(r_{\epsilon}^{\alpha-N}\right) \phi_{R} \\
\leq & (N+1)\left(c_{1} R^{\alpha-4} f_{B_{2 R}} u_{n}^{2}-R^{\alpha} f_{B_{4 R}} h_{n} \phi_{2 R},\right),
\end{aligned}
$$

and concluding again by letting, first $n \rightarrow \infty$, and then $\epsilon \rightarrow 0$.

Proof of (6.40). Applying Theorem (6.1) to (6.42), from (6.1) we deduce that

$$
\int h_{n} \varphi \leq c\left[\int u_{n}^{2} \frac{|\Delta \varphi|^{2}}{\varphi}+\int u_{n}^{2}\left|\Delta \frac{|\nabla \varphi|^{2}}{\varphi}\right|+\int u_{n}^{2} \frac{|\nabla \varphi|^{4}}{\varphi^{3}}\right] .
$$

By letting $n \rightarrow \infty$, we have

$$
\int H(u) \varphi \leq c\left[\int u^{2} \frac{|\Delta \varphi|^{2}}{\varphi}+\int u^{2}\left|\Delta \frac{|\nabla \varphi|^{2}}{\varphi}\right|+\int u^{2} \frac{|\nabla \varphi|^{4}}{\varphi^{3}}\right] .
$$

Now, since

$$
c_{q} \int|u|^{q+1} \varphi \leq \int H(u) \varphi
$$

arguing as in the proof of Theorem 6.1, and using the same chain of inequalities (6.14), we get the estimates (6.40).

Plugging the second estimate of (6.40) in (6.37), we obtain (6.41), concluding the proof.

\subsection{Liouville theorems: distributional solutions}

In this section we shall prove some Liouville theorems within the class of distributional solutions for the problem $\left({ }_{g} \mathbf{P}_{f}\right)$.

Theorem 6.12. Assume that $f, g: \mathbb{R} \rightarrow \mathbb{R}$ are continuous functions satisfying

$$
f(t) t \geq 0, g(t) t \geq 0, \quad \text { for all } t \in \mathbb{R} .
$$

Let $u$ be a distributional solution of

$$
g(u) \geq-\Delta^{2} u \geq f(u) \quad \text { on } \mathbb{R}^{N},
$$


such that $u \in L_{\text {loc }}^{s}\left(\mathbb{R}^{N}\right), 2 \leq s \leq+\infty$ and $g(u), f(u) \in L_{\text {loc }}^{s^{\prime}}\left(\mathbb{R}^{N}\right)$.

Assume that

$$
N=5,6,7 \text { and } \lim _{R \rightarrow \infty} f_{A_{R}}|u|^{2}=0,
$$

or

$$
N \geq 8 \text { and } R^{\alpha_{1}-4} f_{A_{R}}|u|^{2} \leq C, \text { for any R large, }
$$

where $\alpha_{1}$ is defined in (5.37), then $u \equiv 0$ a.e. in $\mathbb{R}^{N}$.

Proof. We shall argue as in the proof of Theorem 6.4. Set $\alpha:=4$ if $N=5,6,7$ and $\alpha:=\alpha_{1}$ in the remaining cases $N \geq 8$.

Step 1. We begin proving the claim under the hypothesis

$$
\lim _{R \rightarrow \infty} R^{\alpha-4} f_{A_{R}}|u|^{2}=0
$$

From (6.37), it follows that

$$
\frac{1}{2} \int_{B_{R}} u^{2} \Delta^{2}\left(r_{\epsilon}^{\alpha-N}\right) \leq c R^{\alpha-4} f_{A_{R / 2} \cup A_{R} \cup A_{2 R}} u^{2} .
$$

Hence, by letting $R \rightarrow+\infty$ we get $\int_{\mathbb{R}^{N}} u^{2} \Delta^{2}\left(r_{\epsilon}^{\alpha-N}\right)=0$, that is $u \equiv 0$ a.e. in $\mathbb{R}^{N}$.

Step 2. If (6.48) holds, from (6.50) by letting $R \rightarrow+\infty$ we have,

$$
\int_{\mathbb{R}^{N}} u^{2} \Delta^{2}\left(r_{\epsilon}^{\alpha-N}\right)<\infty,
$$

which implies

$$
\lim _{R \rightarrow+\infty} \int_{A_{R}} u^{2} \Delta^{2}\left(r_{\epsilon}^{\alpha-N}\right)=0
$$

Now, since in this case $\alpha=\alpha_{1}>4$, and $\Delta^{2}\left(r_{\epsilon}^{\alpha-N}\right) \geq c r^{\alpha-4-N} \geq c R^{\alpha-4-N}$ on $A_{R}$, the hypotheses (6.49) holds and the claim follows from Step 1.

Remark 6.13. If $N=1,2,3,4$, Theorem 6.12 still holds provided

$$
f(t)=0 \text { if and only if } g(t)=0,
$$

and

$$
\lim _{R \rightarrow \infty} f_{A_{R}}|u|^{2}=0
$$

Indeed from (6.36), we have

$$
\int_{B_{R}} H(u) \leq c R^{N-4} f_{A_{R}} u^{2} \rightarrow 0 \text {, as } R \rightarrow \infty .
$$

Hence $f(u) u=0$ a.e. whenever $u \geq 0$ and $g(u) u=0$ a.e. for $u<0$. Therefore $g(u)=f(u)=0$ whenever $u \neq 0$, and since $f$ and $g$ are continuous we deduce that $g(u)=f(u) \equiv 0$ a.e. Since $u$ solves $\left(g \mathbf{P}_{f}\right)$ it follows that $\Delta^{2} u=0$ in distributional sense. Hence by a standard argument $u$ is smooth. Now by using Theorem 6.4 we achieve the claim.

Theorem 6.14. Assume that $f, g: \mathbb{R} \rightarrow \mathbb{R}$ are continuous functions satisfying

$$
f(t) t \geq 0, \quad g(t) t \geq 0, \quad \text { for all } t \in \mathbb{R} .
$$

Let $H$ be defined as (1.6) and assume that

$$
H(t) \geq c_{q}|t|^{q+1}, \quad \text { for all } t \in \mathbb{R}, \text { and for some } q>1, c_{q}>0 .
$$


Let $u$ be a distributional solution of

$$
g(u) \geq-\Delta^{2} u \geq f(u) \quad \text { on } \mathbb{R}^{N},
$$

such that $u \in L_{\text {loc }}^{s}\left(\mathbb{R}^{N}\right), 2 \leq s \leq+\infty$, and $f(u), g(u) \in L_{\text {loc }}^{s^{\prime}}\left(\mathbb{R}^{N}\right)$.

If $N=1, \ldots, 7$ and $q>1$ or

$$
N \geq 8 \text { and } 1<q \leq q_{N},
$$

where $q_{N}$ is defined in (6.26), then $u \equiv 0$ a.e. in $\mathbb{R}^{N}$.

Proof. The proof is similar to the proof of Theorem 6.8. Let $N=1, \ldots, 4$. The claim in this case follows directly form (6.40). Indeed we have,

$$
\int_{B_{R}}|u|^{q+1} \leq C R^{N-4 \frac{q+1}{q-1}} \rightarrow 0 \text { as } R \rightarrow+\infty .
$$

Let $N \geq 5$. From the a priori estimate (6.40) we deduce that

$$
R^{\alpha-4} f_{A_{R}}|u|^{2} \leq R^{\alpha-4 \frac{q+1}{q-1}}
$$

If $N=5,6,7$, the choice $\alpha=4$ is admissible for an the application of Theorem 6.12 and since $4-4 \frac{q+1}{q-1}<0$ the claim follows.

Let $N \geq 8$. In this case, $\alpha=\alpha_{1}>4$ is admissible in Theorem 6.12 and since $\alpha_{1}-4 \frac{q+1}{q-1} \leq 0$ we conclude the proof.

Remark 6.15. Notice that if instead of the double inequality $\left({ }_{g} \mathbf{P}_{f}\right)$ we deal with the one side inequality

$$
-\Delta^{2} u \geq|u|^{q-1} u \text { on } \mathbb{R}^{N},
$$

it is easy to see that (6.52) admits the non trivial solution $u(x)=1-x_{1}^{4} / 24$ for any $q>0$.

This example can be extended to the more general case of the one side inequality

$$
-\Delta^{2} u \geq f(u) \text { on } \mathbb{R}^{N},
$$

with $f \in \mathscr{C}(\mathbb{R})$ satisfying the following assumption: there exists $k>0$ such that

$$
f(t) \leq f(k), \forall t \leq k .
$$

Indeed (6.53) admits the solution $u(x)=k-c|x|^{4}$ with $c>0$ such that $8 N(N+2) c \geq f(k)$. Examples of functions satisfying (6.54) are the nondecreasing functions, $f(t)=|t|^{q-1} t+\gamma \sin t$ for $q>0$ and $\gamma \in \mathbb{R}$ or $f(t)=|t|^{q-1} t \sin t$ for $q>0$.

Corollary 6.16. Let $f, g: \mathbb{R} \rightarrow \mathbb{R}$ are continuous functions. Assume that $H$ satisfies

$$
\begin{aligned}
H(t) & >0 \text { for } t \neq 0, \\
\liminf _{t \rightarrow 0} \frac{H(t)}{t^{2}} & >0(\text { possibly }+\infty), \\
\liminf _{t \rightarrow \pm \infty} \frac{H(t)}{|t|^{p+1}} & >0(\text { possibly }+\infty) \text { for some } p>1 .
\end{aligned}
$$

Let $u$ is a distributional solution of $\left(g \mathbf{P}_{f}\right)$ such that $u \in L_{l o c}^{s}\left(\mathbb{R}^{N}\right), 2 \leq s \leq+\infty$ and $f(u), g(u) \in L_{l o c}^{s^{\prime}}\left(\mathbb{R}^{N}\right)$.

Then $u \equiv 0$ a.e. in $\mathbb{R}^{N}$.

Proof. Let $q>1$ be such that $q \leq \min \left\{p, q_{N}\right\}$ if $N \geq 8$, while $q:=p$ for $N=1, \ldots, 7$.

From the hypotheses on $H$, it follows that $H(t) \geq c_{q}|t|^{q+1}$ for any $t \in \mathbb{R}$ and a suitable $c_{q}>0$. An application of Theorem 6.14 completes the proof. 
Example 6.17. When dealing with the equation

$$
-\Delta^{2} u=f(u) \text { on } \mathbb{R}^{N}
$$

we have that $g=f$ and $H(t)=f(t) t$. Examples of functions $f$ such that the corresponding function $H$ satisfies (6.51) are the following.

- $f(u)=\lambda_{1} u+\lambda_{2}|u|^{p-1} u$ with $\lambda_{1}, \lambda_{2}>0$. In this case $f$ satisfies (6.51) with any $1<q \leq p$, and Corollary 6.16 applies.

- Let $p>1$ and $\lambda>0$, the function $f(u):=u+\sin (u)+\lambda_{2}|u|^{p-1} u$, satisfies (6.51) with any $1<q \leq p$.

- Let $0<p_{1}<p_{2}<p_{3}, \lambda_{1}, \lambda_{2}>0$, and $\mu \in \mathbb{R}$. The functions $f_{1}(u):=\lambda_{1}|u|^{p_{1}-1} u+\mu|u|^{p_{2}-1} u+\lambda_{2}|u|^{p_{3}-1} u$, and $f_{2}(u):=\lambda_{1}|u|^{p_{1}-1} u+\mu|u|^{p_{2}}+\lambda_{2}|u|^{p_{3}-1} u$, satisfy (6.51) with any $p_{1} \leq q \leq p_{3}$, provided $\mu$ is small enough. In particular if $0<p_{1} \leq 1<p_{3}$, then Corollary 6.16 applies.

\subsection{A generalization}

Results similar to Theorem 6.14 can be formulated for more general nonlinearities $f$ and $g$. For instance when the nonlinearity $f$ and $g$ behave differently for positive and negative values of the independent variable, that is when

$$
H(t) \geq\left\{\begin{array}{l}
c_{1}|u|^{q+1}, \quad \text { if } t \geq 0, \\
c_{2}|u|^{p+1}, \quad \text { if } t<0,
\end{array}\right.
$$

with $c_{1}, c_{2}>0$. More generally we have.

Theorem 6.18. Let $f, g: \mathbb{R} \rightarrow \mathbb{R}$ be continuous functions. Assume that $H$ satisfies

$$
H(t) \geq c_{H} \min \left\{|t|^{q+1},|t|^{p+1}\right\}, \forall t \in \mathbb{R} \text {, for some } q \geq p>1,
$$

with $c_{H}>0$. Let $u$ is a distributional solution of $\left({ }_{g} \mathbf{P}_{f}\right)$ such that $u \in L_{l o c}^{s}\left(\mathbb{R}^{N}\right), 2 \leq s \leq+\infty$ and $f(u), g(u) \in$ $L_{\text {loc }}^{s^{\prime}}\left(\mathbb{R}^{N}\right)$.

Then for large $R>0,(6.40)$ and (6.41) hold.

Moreover, if $N=1, \ldots, 7$ or $N \geq 8$ and $1<q \leq q_{N}$, then $u \equiv 0$ a.e. in $\mathbb{R}^{N}$.

Proof. Let

$$
h(u):=\min \left\{|u|^{q+1},|u|^{p+1}\right\}= \begin{cases}|u|^{q+1} & \text { if }|u| \leq 1, \\ |u|^{p+1} & \text { if }|u|>1 .\end{cases}
$$

Arguing as in the proof of Theorem 6.11 we obtain inequality (6.47), which in turn yields

$$
c_{H} \int h(u) \varphi \leq \int_{\mathbb{R}^{N}} H(u) \varphi \leq c\left[\int u^{2} \frac{|\Delta \varphi|^{2}}{\varphi}+\int u^{2}\left|\Delta \frac{|\nabla \varphi|^{2}}{\varphi}\right|+\int u^{2} \frac{|\nabla \varphi|^{4}}{\varphi^{3}}\right] .
$$

Next denoting with $\chi_{1}$ and $\chi_{2}$ the characteristic functions of $\Omega_{1}:=\{x:|u(x)| \leq 1\}$ and $\Omega_{2}:=\{x:|u(x)|>1\}$ respectively, we have $h(u)=|u|^{q+1} \chi_{1}+|u|^{p+1} \chi_{2}$. Arguing as in the proof of (6.2), and with the same notation, we observe that each term in the right hand side of (6.56) has the form (6.13). By using Hölder and Young inequalities, with exponents $x=\frac{q+1}{2}$ and $y=\frac{p+1}{2}$ and parameter $\epsilon$ and $\delta$, we have

$$
\begin{aligned}
& \int_{\mathbb{R}^{N}} H(u) \varphi \leq c \int_{A_{R}} u^{2} \chi_{1}|\psi|+\int_{A_{R}} u^{2} \chi_{2}|\psi| \\
& \quad \leq\left(\int|u|^{q+1} \chi_{1} \varphi\right)^{\frac{1}{x}}\left(\int \frac{|\psi|^{\chi^{\prime}}}{\varphi^{\chi^{\prime}-1}}\right)^{\frac{1}{\chi^{\prime}}}+\left(\int|u|^{p+1} \chi_{2} \varphi\right)^{\frac{1}{y}}\left(\int \frac{|\psi|^{y^{\prime}}}{\varphi^{y^{\prime}-1}}\right)^{\frac{1}{y^{\prime}}}
\end{aligned}
$$




$$
\begin{aligned}
& \leq \frac{\epsilon^{x}}{x c_{1}} \int|u|^{q+1} \chi_{1} \varphi+\frac{1}{x^{\prime} \epsilon^{x^{\prime}}} \int \frac{|\psi|^{x^{\prime}}}{\varphi^{x^{\prime}-1}}+\frac{\delta^{y}}{y c_{1}} \int|u|^{p+1} \chi_{2} \varphi+\frac{1}{y^{\prime} \delta^{y^{\prime}}} \int \frac{|\psi|^{y^{\prime}}}{\varphi^{y^{\prime}-1}} \\
& \leq\left(\frac{\epsilon^{x}}{x c_{1}}+\frac{\delta^{y}}{y c_{1}}\right) \int h(u) \varphi+\frac{1}{x^{\prime} \epsilon^{x^{\prime}}} \int \frac{|\psi|^{\chi^{\prime}}}{\varphi^{x^{\prime}-1}}+\frac{1}{y^{\prime} \delta^{y^{\prime}}} \int \frac{|\psi|^{y^{\prime}}}{\varphi^{y^{\prime}-1}} .
\end{aligned}
$$

Taking $\varphi:=\phi_{R}=\phi_{1}(|x| / R)$, and with a suitable choice of $\epsilon$ and $\delta$, since $q \geq p$, we have

$$
\int_{\mathbb{R}^{N}} H(u) \varphi \leq c R^{N-4 \frac{q+1}{q-1}}+c R^{N-4 \frac{p+1}{p-1}} \leq c R^{N-4 \frac{q+1}{q-1}},
$$

which is the first estimate in (6.40). To obtain the second estimate in (6.40), by Hölder inequality and estimate (6.57), we argue as follows

$$
\begin{aligned}
\left(f_{B_{R}} u^{2}\right)^{1 / 2} & \leq\left(f_{B_{R}} u^{2} \chi_{1}\right)^{1 / 2}+\left(f_{B_{R}} u^{2} \chi_{2}\right)^{1 / 2} \\
& \leq\left(f_{B_{R}}|u|^{q+1} \chi_{1}\right)^{\frac{1}{q+1}}+\left(f_{B_{R}}|u|^{p+1} \chi_{2}\right)^{\frac{1}{p+1}} \\
& \leq\left(\frac{1}{c_{f}} f_{B_{R}} H(u)\right)^{\frac{1}{q+1}}+\left(\frac{1}{c_{f}} f_{B_{R}} H(u)\right)^{\frac{1}{p+1}} \\
& \leq c R^{-4 \frac{1}{q-1}}+c R^{-\frac{4}{p+1} \frac{q+1}{q-1}} \leq c R^{-4 \frac{1}{q-1}} .
\end{aligned}
$$

Finally, the claim follows arguing as in the proof of Theorem 6.14 .

\section{Further remarks and results on the solutions}

The main purpose of this Section is to show further qualitative properties on the possible solutions of our prototype equation (1.3) and for more general problems. In order to simplify the presentation we consider only smooth solutions.

\subsection{Representation formula for $\boldsymbol{u}^{2}$}

Theorem 7.1. Let $h \in \mathscr{C}\left(\mathbb{R}^{N}\right)$ and $u \in \mathscr{C}^{4}\left(\mathbb{R}^{N}\right)$ be such that $-u \Delta^{2} u=h$. Let $x \in \mathbb{R}^{N}$.

The representation formula

$$
\begin{aligned}
-\frac{C_{N}}{2} u^{2}(x)= & \int_{\mathbb{R}^{N}} \frac{h(y)}{|x-y|^{N-4}} d y+\int_{\mathbb{R}^{N}} \frac{|\Delta u|^{2}(y)}{|x-y|^{N-4}} d y \\
& +2(N-4) \int_{\mathbb{R}^{N}} \frac{|\nabla u|^{2}(y)}{|x-y|^{N-2}} d y \\
& -2(N-4)(N-2) \int_{\mathbb{R}^{N}} \frac{(x-y \cdot \nabla u(y))^{2}}{|x-y|^{N}} d y,
\end{aligned}
$$

holds ${ }^{2}$ provided one of the following assumptions is satisfied

1. $h \geq 0$,

$$
\lim _{R} f_{R<|x-y|<2 R} u^{2}(y) d y=0
$$

$2 C_{N}$ is the normalization constant defined in (1.9). 
and

$$
\int_{\mathbb{R}^{N}} \frac{|\nabla u|^{2}}{|x|^{N-2}}<\infty
$$

2. (7.2) holds and

$$
\int_{\mathbb{R}^{N}} \frac{|h|}{|X|^{N-4}}<+\infty, \int_{\mathbb{R}^{N}} \frac{(\Delta u)^{2}}{|x|^{N-4}}<+\infty, \text { and } \int_{\mathbb{R}^{N}} \frac{|\nabla u|^{2}}{|x|^{N-2}}<\infty .
$$

3. $-\Delta^{2} u=f(u)$ with $f(u) u \geq c_{q}|u|^{q+1}$ for some $q>1$ and $c_{q}>0$.

Moreover, if one of claims 1., 2. or 3. holds, then all the integrals in the representation formula (7.1) are finite.

Proof. By translation, it suffices to prove the claim for $x=0$. From (5.31) of Proposition 5.13 with $\alpha=4$ and $\varphi=\varphi_{R}=\phi_{R}^{4}$, we have

$$
\begin{aligned}
& \int \frac{h}{|x|^{N-4}} \varphi_{R}+\int(\Delta u)^{2} \frac{\varphi_{R}}{|x|^{N-4}}+ \\
& \quad+2(N-4) \int|\nabla u|^{2} \frac{\varphi_{R}}{|x|^{N-2}}+\frac{C_{N}}{2} u^{2}(0) \varphi_{R}(0) \\
& =2(N-4)(N-2) \int\left(\nabla u \cdot \frac{x}{|x|}\right)^{2} \frac{\varphi_{R}}{|x|^{N-2}} \\
& \quad-2(N-4) E_{1}\left(\nabla u,|x|^{3-N}, \varphi_{R}^{\prime}\right)-E_{2}\left(u,|x|^{4-N}, \varphi_{R}\right) .
\end{aligned}
$$

The representation (7.1) will follows letting $R \rightarrow \infty$ and showing that $E_{1}\left(\nabla u,|x|^{3-N}, \varphi_{R}^{\prime}\right) \rightarrow 0$ and $E_{2}\left(u,|x|^{4-N}, \varphi_{R}\right) \rightarrow 0$.

1. Estimates (6.30) and (6.32), by hypothesis (7.2) and $h \geq 0$, assure that $E_{1}\left(\nabla u,|x|^{3-N}, \varphi_{R}^{\prime}\right) \rightarrow 0$ and $E_{2}\left(u,|x|^{4-N}, \varphi_{R}\right) \rightarrow 0$ as $R \rightarrow \infty$. Furthermore, all the integrals in (7.5) have a limit as $R \rightarrow \infty$ by monotone convergence theorem. All the integrals are finite since all of them are nonnegative and the integral in right hand side of (7.5) is convergent because of (7.3).

2. From the hypotheses, we deduce

$$
\lim _{R} R^{2} f_{A_{R}}|\nabla u|^{2}=0, \text { and } \lim _{R} R^{4} f_{A_{R}}|\Delta u|^{2}=0
$$

Plugging this information in (5.4) and (5.29), we deduce respectively that $E_{1} \rightarrow 0$ and $E_{2} \rightarrow 0$, concluding the proof.

3. Arguing as in the case 1. from the estimates in Theorem 6.1 we deduce that $E_{1} \rightarrow 0$ and $E_{2} \rightarrow 0$, and the representation (7.1) holds. It remains to prove that the integrals are finite.

Plugging the estimate (6.40) in (6.7), we obtain

$$
R^{2} f_{A_{R}}|\nabla u|^{2} \leq c R^{-\frac{8}{q-1}}
$$

which in turn yields

$$
\int_{B_{2 R} \backslash B_{R}} \frac{|\nabla u|^{2}}{|x|^{N-2}} \leq \omega_{N}\left(2^{N}-1\right) R^{2} f_{A_{R}}|\nabla u|^{2} \leq c \omega_{N}\left(2^{N}-1\right) R^{-\frac{8}{q-1}} .
$$

Let $k \geq 1$, we have

$$
\int_{B_{2^{k+1}}} \frac{|\nabla u|^{2}}{|x|^{N-2}}=\int_{B_{1}} \frac{|\nabla u|^{2}}{|x|^{N-2}}+\sum_{j=0}^{k} \int_{B_{2^{j+1}} \backslash B_{2^{j}}} \frac{|\nabla u|^{2}}{|x|^{N-2}}
$$




$$
\begin{aligned}
& \leq \int_{B_{1}} \frac{|\nabla u|^{2}}{|x|^{N-2}}+c \sum_{j=0}^{k}\left(2^{j}\right)^{-\frac{8}{q-1}} \\
& <\int_{B_{1}} \frac{|\nabla u|^{2}}{|x|^{N-2}}+\frac{c}{1-2^{-\frac{8}{q-1}}}<\infty .
\end{aligned}
$$

By letting $k \rightarrow \infty$, it follows that (7.3) holds.

The following Lemma, which we believe is interesting in itself, provides a sufficient condition for the validity of (7.4).

Lemma 7.2. Let $h \in L_{\text {loc }}^{1}\left(\mathbb{R}^{N}\right)$ be a nonnegative function and let $u \in H_{\text {loc }}^{2}\left(\mathbb{R}^{N}\right)$ be a solution of $\left(\mathbf{P}_{h}\right)$. Assume that there exist $\theta:] 0,+\infty[\rightarrow] 0,+\infty[$ be such that for $R>0$ large there holds

$$
f_{A_{R}} u^{2} \leq \theta(R) \quad \text { for } R \text { large. }
$$

If $\sum_{j=0}^{\infty} \theta\left(2^{j}\right)<\infty$, then

$$
\frac{h}{|x|^{N-4}}, \frac{(\Delta u)^{2}}{|\chi|^{N-4}}, \frac{|\nabla u|^{2}}{|x|^{N-2}} \in L^{1}\left(\mathbb{R}^{N} \backslash B_{1}\right)
$$

Moreover

1. If $h \in L_{\text {loc }}^{\infty}$, we have $\frac{h}{|x|^{N-4}}, \in L^{1}\left(\mathbb{R}^{N}\right)$.

2. If $u \in \mathscr{C}^{4}\left(\mathbb{R}^{N}\right)$, then the functions in (7.7) belong to $L^{1}\left(\mathbb{R}^{N}\right)$.

Remark 7.3. Special cases of solutions of $\left(\mathbf{P}_{h}\right)$ that fulfill the hypotheses of the above Lemma are the following. 1. By simple computation it is easy to see that if there exist $c, \sigma>0$ such that

$$
f_{A_{R}} u^{2} \leq c R^{-\sigma}
$$

then Lemma 7.2 applies and (7.7) holds.

2. From the a priori estimates in Theorem (6.8) and (6.9), it is immediate to verify that if $h \geq c|u|^{q+1}$ with $q>1$ and $c>0$, then Lemma 7.2 applies and (7.7) holds and $\frac{|u|^{q+1}}{1+|x|^{N-4}} \in L^{1}\left(\mathbb{R}^{N}\right)$.

Proof. Let us prove that $\frac{h}{|x|^{N-4}} \in L^{1}\left(\mathbb{R}^{N} \backslash B_{1}\right)$. The proofs of the other claims follow similarly.

Since $\frac{h}{|x|^{N-4}}$ is nonnegative it is enough to show that $R \mapsto \int_{B_{R} \backslash B_{1}} \frac{h}{1+|x|^{N-4}}$ is bounded for a subsequence $R_{k} \rightarrow \infty$.

To this end we choose $R_{k}:=2^{k+1}$. We have,

$$
\int_{B_{R_{k}} \backslash B_{1}} \frac{h}{|x|^{N-4}}=\sum_{j=0}^{k} \int_{B_{2^{j+1}} \backslash B_{2^{j}}} \frac{h}{|x|^{N-4}} .
$$

Now, since by using (6.5) and the assumption (7.6) each addendum of the right hand side of the above identity can be estimate as

$$
\int_{B_{2 R} \backslash B_{R}} \frac{h}{|x|^{N-4}} \leq \frac{\omega_{N} R^{4}}{\left|B_{2 R}\right|} \int_{B_{2 R} \backslash B_{R}} h \leq \omega_{N} c_{1} f_{A_{2 R}} u^{2} \leq c \theta(2 R),
$$

it follows that

$$
\int_{B_{R_{k}} \backslash B_{1}} \frac{h}{|x|^{N-4}} \leq \sum_{j=0}^{k} c \theta\left(2^{j+1}\right) \leq c \sum_{j=0}^{\infty} \theta\left(2^{j}\right)<\infty .
$$

This completes the proof of the first claim in (7.7).

The proof of other cases follows by using similar argument and the estimates (6.5) and (6.7). 


\subsection{Remarks on the sign of the solutions}

Theorem 7.4. Let $u \in \mathscr{C}^{4}\left(\mathbb{R}^{N}\right)$ be a solution of $\left({ }_{g} \mathbf{P}_{f}\right)$ with $f$, $g$ continuous functions and $H$ defined as in (1.6) and satisfying (6.55). If one of the following condition holds

1. u has a sign;

2. $\Delta u$ has a sign;

3. $u \Delta u$ has a sign;

then $u \equiv 0$ in $\mathbb{R}^{N}$.

Proof. Clearly the interesting cases occour for $N \geq 8$. From Theorem 6.18, we know that (6.40) holds. Plugging (6.40) in (6.2) and since the problem is invariant by translation, we deduce

$$
\lim _{R} f_{B_{R}(x)}|u|^{2}=0, \quad \text { and } \lim _{R} f_{B_{R}(x)}(\Delta u)^{2}=0, \quad \text { for any } x \in \mathbb{R} .
$$

Which in turns implies

$$
\liminf _{R} f_{B_{R}(x)}|u|=0 .
$$

The above limits will play a crucial role to get information on the sign of the solutions.

1. Without loss of generality we may assume that $u \geq 0$. In this case $u$ solves

$$
-\Delta^{2} u \geq f(u) \geq c_{H} \min \left\{|u|^{q},|u|^{p}\right\} \geq 0 .
$$

Arguing as in the proof of Theorem 3.1, since $-u$ satisfies the ring condition (7.10), from the representation formula of Theorem A.1, it follows that $u \leq 0 \leq u$. Hence $u \equiv 0$ in $\mathbb{R}^{N}$.

In the case $u \leq 0$ we deduce that $-u$ solves

$$
-\Delta^{2}(-u) \geq-g(u) \geq c_{H} \min \left\{|u|^{q},|u|^{p}\right\} \geq 0,
$$

which by the same argument above yields $-u \leq 0$.

2. Without loss of generality we assume that $-\Delta u \geq 0$. Since $u$ is superharmonic and satisfies the ring condition (7.10), arguing as in the proof of Theorem 3.1, by the representation formula of Theorem A.1, it follows that $u$ is nonnegative and by point 1 . we get the claim (the case $\Delta u \geq 0$ can be handled similarly).

3. First we consider the case $u \Delta u \geq 0$. Indeed, by (6.10) with $\Phi=u$ we have that $\Delta u^{2} \geq 0$. Since $u^{2}$ satisfies (7.9), by the representation formula of Theorem A.1, we obtain that $u^{2} \leq 0$ and hence the claim.

Assume that $-u \Delta u \geq 0$. Observing that $-u,-f(u),-g(u)$ and $\Delta^{2} u$ have the same sign, we deduce that $\Delta^{2} u \Delta u \geq 0$. Therefore from (6.10) with $\Phi=\Delta u$ we have that $\Delta(\Delta u)^{2} \geq 0$. Since $(\Delta u)^{2}$ satisfies the ring condition (7.9), again by the the representation formula of Theorem A.1, we deduce $\Delta u=0$ that is $u$ is harmonic, which implies that $u \equiv 0$ because $u$ satisfies the ring condition (7.10).

The statements 1. and 2. of Theorem 7.4 and their proofs still hold for the higher order problem

$$
(-\Delta)^{m} u+|u|^{q-1} u=0 \quad \text { on } \mathbb{R}^{N},
$$

as well as for its generalizations in the same spirit of $\left({ }_{g} \mathbf{P}_{f}\right)$.

\subsection{Uniqueness}

Theorem 7.5. Let $f \in \mathscr{C}(\mathbb{R})$ be a continuous function such that

$$
(f(t)-f(s))(t-s) \geq c_{f}|t-s|^{q+1}, \quad \forall t, s \in \mathbb{R},
$$




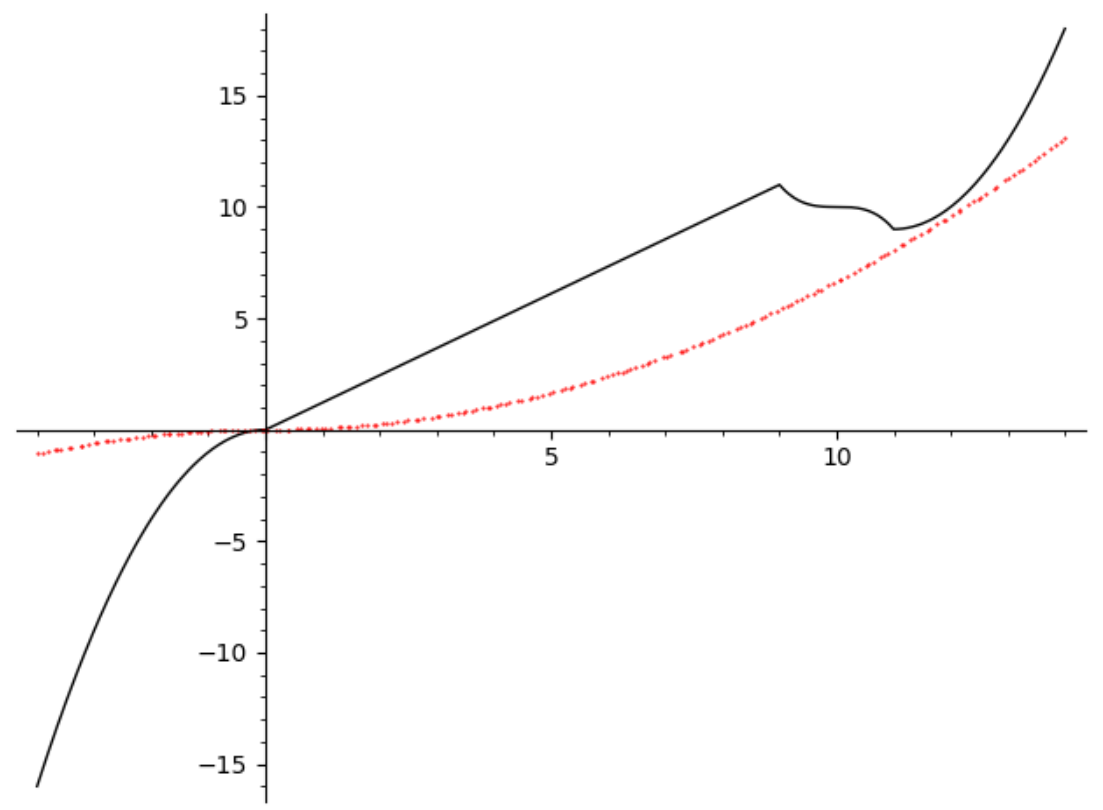

Fig. 1: $f(t)$ in continuous line, $c|t|^{q-1} t$ in dotted line.

for some $q>1$ and $c_{f}>0$. Let $k \in \mathscr{C}\left(\mathbb{R}^{N}\right)$ and let $u \in \mathscr{C}^{4}\left(\mathbb{R}^{N}\right)$ be a solution of

$$
-\Delta^{2} u=f(u)+k \text { on } \mathbb{R}^{N} .
$$

If $N=1, \ldots, 7$ or $N \geq 8$ and $1<q \leq q_{N}$, where $q_{N}$ is defined in (6.26), then $u$ is unique.

Proof. Let $u$ and $v$ be solutions of (7.13) and set $w:=u-v$. We have that $w$ solves the problem

$$
\begin{aligned}
-\Delta^{2} w w & =\left(\left(-\Delta^{2} u\right)-\left(-\Delta^{2} v\right)\right)(u-v)=(f(u)-f(v))(u-v) \\
& \geq c_{f}|u-v|^{q+1}=c_{f}|w|^{q+1} .
\end{aligned}
$$

From Theorem 6.8 we get the claim.

Remark 7.6. Condition (7.12) implies that $f$ is increasing. Without the increasing property of $f$, the uniqueness results is in general false. Indeed, let $f$ be defined as

$$
f(t):= \begin{cases}\frac{11}{9} t, & \text { if } 0<t<9, \\ 10-|t-10|^{\frac{N+4}{N-4}-1}(t-10), & \text { if } 9 \leq t \leq 11, \\ 9+(t-11)^{q}, & \text { if } t>11, \\ -|t|^{q}, & \text { if } t \leq 0,\end{cases}
$$

where $q$ is any number $q>1$. See Figure 1. Clearly this $f$ is not increasing and hence (7.12) does not hold, while $f$ satisfies

$$
f(t) t \geq c_{q}|t|^{q+1}, \quad \forall t \in \mathbb{R} \text {, and } c_{q}>0 \text { sufficiently small. }
$$

The problem

$$
-\Delta^{2} u=f(u)-10 \text {, on } \mathbb{R}^{N}, N>4,
$$

admits the constants solution $u(x)=10$ as well as the function

$$
v(x)=10+\frac{c}{\left(\epsilon^{2}+|x|^{2}\right)^{\frac{N-4}{2}}}
$$

for suitable $\epsilon, c>0\left(\right.$ namely, $c^{8 /(N-4)}=(N-4)\left(N^{2}-4\right) N \epsilon^{4}$ and $\left.\epsilon^{4} \geq(N-4)\left(N^{2}-4\right) N\right)$. 
Remark 7.7 (On the symmetry preserving property). As usual the uniqueness result implies several symmetry properties on the solutions of (7.13). For instance, assuming that $f$ satisfies the hypotheses in the uniqueness result Theorem 7.5, we have

- if $k \in \mathscr{G}\left(\mathbb{R}^{N}\right)$ is a radial function, then the solution of (7.13) is radial;

- if $k \in \mathscr{C}\left(\mathbb{R}^{N}\right)$ is even is some direction, then the solution of (7.13) shares the same symmetry;

- if $k \in \mathscr{C}\left(\mathbb{R}^{N}\right)$ depends only on $j<N$ variables, say $x_{1}, \ldots, x_{j}$, then also the solution of (7.13) depends only on $x_{1}, \ldots, x_{j}$.

Remark 7.8 (On the sign preserving property). The prototype case related to (7.13) for the second order case is

$$
\Delta u=|u|^{q-1} u-k .
$$

In this case the problem present a sign preserving property, namely, if $k$ is nonnegative then also the solution is nonnegative. See for instance [9] where a discussion of the quasilinear case is presented.

For the higher order case this property cannot be expected (in general the maximum principle fails and a Kato's inequality does not hold). Indeed, for instance, consider the problem

$$
-\Delta^{2} u=|u|^{q-1} u-k,
$$

and for simplicity consider the 1-dimensional case $N=1$ (by a lifting argument our examples are still valid in higher dimension). Choosing $k(x)=1$, the only solution of (7.14) is the constant function $u(x)=1$, which has the same sign of $k$. While by choosing $k(x)=\left|x^{4}-1\right|^{q-1}\left(x^{4}-1\right)+24$, which is positive, it follows that (7.14) is solved by the changing sign function $u(x)=x^{4}-1$.

\section{A Representation formula}

Here we state some results from [6] for the reader convenience. The main equation is

$$
(-\Delta)^{m} u=\mu \quad \text { on } \mathbb{R}^{N}
$$

where $\mu$ is a positive Radon measure.

Theorem A.1. Let $m \geq 1$ be an integer and $N>2 m$. Let $\mu$ be a positive Radon measure on $\mathbb{R}^{N}$ and $l \in \mathbb{R}$. The following statements are equivalent:

a) $u$ is a distributional solution of (A.1) and for a.e. $x \in \mathbb{R}^{N}$,

$$
\liminf _{R \rightarrow+\infty} \frac{1}{R^{N}} \int_{R \leq|x-y| \leq 2 R}|u(y)-l| d y=0 .
$$

b) $u$ is a distributional solution of (A.1), essinf $u=l$ and $u$ is weakly superharmonic.

c) $u \in L_{\text {loc }}^{1}\left(\mathbb{R}^{N}\right)$ and we have

$$
u(x)=l+c(2 m) \int_{\mathbb{R}^{N}} \frac{d \mu(y)}{|x-y|^{N-2 m}} \quad \text { a.e. } x \in \mathbb{R}^{N},
$$

where, for general $\alpha>0$ with $0<\alpha<N, c(\alpha):=\frac{\Gamma\left(\frac{N-\alpha}{2}\right)}{2^{\alpha} \pi^{N / 2} \Gamma\left(\frac{\alpha}{2}\right)}$.

Moreover, if $a), b$ ) or $c$ ) holds, then for $i=1, \ldots, m$ the distribution $(-\Delta)^{i} u$ is a positive Radon measure and can be represented by

$$
\left\langle(-\Delta)^{i} u, \varphi\right\rangle=\int_{\mathbb{R}^{N}} u(-\Delta)^{i} \varphi=c(2(m-i)) \int_{\mathbb{R}^{N}} \varphi(x) \int_{\mathbb{R}^{N}} \frac{d \mu(y)}{|x-y|^{N-2(m-i)}} d x .
$$


Acknowledgments: The authors thank the referees for the careful reading of the manuscript and for suggesting the possible connections with the papers [3], [28], [29], as well as with [14]. The authors acknowledge the support of FRA 2018 Università di Trieste.

Conflict of interest: Authors state no conflict of interest.

\section{References}

[1] L. Ambrosio and X. Cabré, Entire solutions of semilinear elliptic equations in $\mathbb{R}^{3}$ and a conjecture of De Giorgi, J. Amer. Math. Soc. 13 (2000), no. 4, 725-739.

[2] F. Bernis, Elliptic and parabolic semilinear problems without conditions at infinity, Arch. Ration. Mech. Anal. 106 (1989), 3, 217-241.

[3] B. Bianchini, G. Colombo, M. Magliaro, L. Mari, P. Pucci and M. Rigoli, Recent rigidity results for graphs with prescribed mean curvature, Math. Eng. 3 (2021), no. 5, Paper No. 039, 1-48.

[4] B. Bianchini, L. Mari, P. Pucci and M. Rigoli, Geometric analysis of quasilinear inequalities on complete manifolds-maximum and compact support principles and detours on manifolds. Frontiers in Mathematics. Birkhäuser/Springer, Cham, 2021, $x+286$ pp.

[5] H. Brezis, Semilinear equations in $\mathbb{R}^{n}$ without condition at infinity, Appl. Math. Optim. 12 (1984), 271-282.

[6] G. Caristi, L. D’Ambrosio and E. Mitidieri, Representation formulae for solutions to some classes of higher order systems and related Liouville theorems, Milan J. Math. 76 (2008), 27-67.

[7] S.-H. A. Chang, On a fourth-order partial differential equation in conformal geometry. Harmonic analysis and partial differential equations (Chicago, IL, 1996), 127-150, Chicago Lectures in Math., Univ. Chicago Press, Chicago, IL, 1999.

[8] S.-H. A. Chang and P.C. Yang, On a fourth order curvature invariant. Spectral problems in geometry and arithmetic (lowa City, IA, 1997), 9-28, Contemp. Math., 237, Amer. Math. Soc., Providence, RI, 1999.

[9] L. D’Ambrosio, A. Farina, E. Mitidieri and J. Serrin, Comparison principles, uniqueness and symmetry results of solutions of quasilinear elliptic equations and inequalities, Nonlinear Anal. 90 (2013), 135-158.

[10] L. D’Ambrosio and E. Mitidieri, A priori estimates, positivity results, and nonexistence theorems for quasilinear degenerate elliptic inequalities, Adv. Math. 224 (2010), no. 3, 967-1020.

[11] L. D'Ambrosio and E. Mitidieri, Nonnegative solutions of some quasilinear elliptic inequalities and applications, (Russian) Mat. Sb. 201 (2010), no. 6, 75-92; translation in Sb. Math. 201 (2010), no. 5-6, 855-871.

[12] L. D’Ambrosio and E. Mitidieri, A priori estimates and reduction principles for quasilinear elliptic problems and applications, Adv. Differential Equations 17 (2012), no. 9-10, 935-1000.

[13] L. D'Ambrosio and E. Mitidieri, Uniqueness and comparison principles for semilinear equations and inequalities in Carnot groups, Adv. Nonlinear Anal. 7 (2018), no. 3, 313-325.

[14] D.E. Edmunds, D. Fortunato and E. Jannelli, Fourth-order nonlinear elliptic equations with critical growth. Atti Accad. Naz. Lincei Rend. Cl. Sci. Fis. Mat. Nat. (8) 83 (1989), 115-119.

[15] A. Farina and J. Serrin, Entire solutions of completely coercive quasilinear elliptic equations, J. Differential Equations 250 (2011), no. 12, 4367-4408.

[16] A. Farina and J. Serrin, Entire solutions of completely coercive quasilinear elliptic equations II, J. Differential Equations 250 (2011), no. 12, 4409-4436.

[17] F. Gazzola, Mathematical models for suspension bridges. Nonlinear structural instability. MS A. Modeling, Simulation and Applications, 15. Springer, Cham, 2015. xxii+259 pp.

[18] F. Gazzola and H.-C. Grunau, Radial entire solutions for supercritical biharmonic equations, Math. Ann. 334 (2008), $905-$ 936.

[19] F. Gazzola, H.-C. Grunau and G. Sweers, Polyharmonic boundary value problems. Positivity preserving and nonlinear higher order elliptic equations in bounded domains. Lecture Notes in Mathematics, 1991. Springer-Verlag, Berlin, 2010. xviii+423 pp.

[20] Z. Huo and Y. Jia, Well-posedness for the fourth-order nonlinear derivative Schrödinger equation in higher dimension, J. Math. Pures Appl. (9) 96 (2011), no. 2, 190-206.

[21] Yan Yan Li, Remark on some conformally invariant integral equations: the method of moving spheres, J. Eur. Math. Soc. 6 (2004), 153-180.

[22] V.G. Maz'ya, Behaviour of solutions to the dirichlet problem for the biharmonic operator at a boundary point. In: Fábera J. (eds) Equadiff IV. Lecture Notes in Mathematics, vol 703 (1979). Springer, Berlin, Heidelberg.

[23] V.G. Maz'ya, The Wiener test for higher order elliptic equations, Duke Math. J. 115 (2002), 479-512.

[24] C. Miao, G. Xu and L. Zhao, Global well-posedness and scattering for the defocusing energy-critical nonlinear Schrödinger equations of fourth order in dimensions $d \geq 9$, J. Differential Equations 251 (2011), no. 12, 3381-3402.

[25] E. Mitidieri, A Rellich type identity and applications, Comm. Partial Differential Equations 18 (1993), no. 1-2, $125-151$. 
[26] E. Mitidieri, A simple approach to Hardy inequalities. (Russian) Mat. Zametki 67 (2000), no. 4, 563-572; translation in Math. Notes 67 (2000), no. 3-4, 479-486.

[27] E. Mitidieri, and S.I. Pohozaev, A priori estimates and the absence of solutions of nonlinear partial differential equations and inequalities, Proc. Steklov Inst. Math. 234 (2001), 1-384.

[28] P. Pucci and J. Serrin, Critical exponents and critical dimensions for polyharmonic operators. J. Math. Pures Appl. (9) 69 (1990), no. 1, 55-83.

[29] P. Pucci and J. Serrin, A remark on entire solutions of quasilinear elliptic equations. J. Differential Equations 250 (2011), no. 2, 675-689.

[30] F. Rellich, Halbbeschränkte Differentialoperatoren höherer Ordnung. In Gerretsen, J.C.H., de Groot, J. (eds.) Proceedings of the International Congress of Mathematicians 1954, vol. III, pp.243-250. Noordhoff, Groningen (1956).

[31] M. Ruzhansky, B. Wang and H. Zhang, Global well-posedness and scattering for the fourth order nonlinear Schrödinger equations with small data in modulation and Sobolev spaces, J. Math. Pures Appl. (9) 105 (2016), no. 1, 31-65.

[32] J. Serrin and H. Zou, Existence of positive entire solutions of elliptic hamiltonian systems, Comm. Partial Differential Equations, 23 (1998), 375-398.

[33] A. Tertikas and N.B. Zographopoulos, Best constants in the Hardy-Rellich inequalities and related improvements, $A d v$. Math. 209 (2007), 407-459. 